\title{
Exploring smoking-gun signals of the Schwinger mechanism in QCD
}

\author{
A. C. Aguilar $\odot,{ }^{1}$ M. N. Ferreira $\odot,{ }^{1}$ and J. Papavassiliou $\odot^{2}$ \\ ${ }^{1}$ University of Campinas-UNICAMP, Institute of Physics "Gleb Wataghin", \\ 13083-859 Campinas, São Paulo, Brazil \\ ${ }^{2}$ Department of Theoretical Physics and IFIC, University of Valencia and CSIC, E-46100 Valencia, Spain
}

(Received 22 November 2021; accepted 10 January 2022; published 27 January 2022)

\begin{abstract}
In Quantum Chromodynamics, the Schwinger mechanism endows the gluons with an effective mass through the dynamical formation of massless bound-state poles that are longitudinally coupled. The presence of these poles affects profoundly the infrared properties of the interaction vertices, inducing crucial modifications to their fundamental Ward identities. Within this general framework, we present a detailed derivation of the non-Abelian Ward identity obeyed by the pole-free part of the three-gluon vertex in the softgluon limit, and determine the smoking-gun displacement that the onset of the Schwinger mechanism produces to the standard result. Quite importantly, the quantity that describes this distinctive feature coincides formally with the bound-state wave function that controls the massless pole formation. Consequently, this signal may be computed in two independent ways: by solving an approximate version of the pertinent BetheSalpeter integral equation, or by appropriately combining the elements that enter in the aforementioned Ward identity. For the implementation of both methods we employ two- and three-point correlation functions obtained from recent lattice simulations, and a partial derivative of the ghost-gluon kernel, which is computed from the corresponding Schwinger-Dyson equation. Our analysis reveals an excellent coincidence between the results obtained through either method, providing a highly nontrivial self-consistency check for the entire approach. When compared to the null hypothesis, where the Schwinger mechanism is assumed to be inactive, the statistical significance of the resulting signal is estimated to be 3 standard deviations.
\end{abstract}

DOI: 10.1103/PhysRevD.105.014030

\section{INTRODUCTION}

The systematic study of the fundamental $n$-point correlation (Green's) functions, such as propagators and vertices, forms an essential element in the ongoing quest for unraveling the nonperturbative properties and underlying dynamical mechanisms of Quantum Chromodynamics (QCD) [1]. In recent years, this challenging problem has been tackled by means of approaches formulated in the continuum, such as the Schwinger-Dyson equations (SDEs) [2-12] or the functional renormalization group [13-17], in conjunction with numerous gauge-fixed lattice simulations [18-27]. This intense activity has delivered new insights on the nature and phenomenology of the strong interactions and has broadened our basic understanding of non-Abelian gauge theories [28-48].

In this context, the characteristic feature of infrared saturation displayed by the gluon propagator has attracted particular attention, being often linked with the emergence

Published by the American Physical Society under the terms of the Creative Commons Attribution 4.0 International license. Further distribution of this work must maintain attribution to the author(s) and the published article's title, journal citation, and DOI. Funded by SCOAP . of a mass gap in the gauge sector of QCD [49-67]. This property has been explored both in large-volume simulations [18-24], and in various functional approaches [61-73], and is rather general, manifesting itself in the Landau gauge, away from it [74-80], and in the presence of dynamical quarks [81-85]. In general terms, the scalar form factor, $\Delta\left(q^{2}\right)$, of the gluon propagator reaches a finite nonvanishing value in the deep infrared, and the gluon mass, $m$, is identified as $\Delta^{-1}(0)=m^{2}$.

One of the nonperturbative mechanisms put forth in order to explain this special behavior of the gluon propagator is based on a non-Abelian extension of the wellknown Schwinger mechanism [86,87]. According to the fundamental observation underlying this mechanism, if the self-energy develops a pole at zero momentum transfer $\left(q^{2}=0\right)$, then the corresponding vector meson (gluon) acquires a mass, even if the gauge symmetry forbids a mass term at the level of the fundamental Lagrangian [86-89].

The precise implementation of this idea at the level of the SDE describing the momentum evolution of $\Delta\left(q^{2}\right)$ requires the inclusion of longitudinally coupled massless poles at the level of the fundamental interaction vertices of the theory [90-94]. These poles are produced as massless bound state excitations, whose formation is governed by a special set of Bethe-Salpeter equations (BSEs) [90-93]. In addition, their presence is crucial for maintaining intact the 
form of the Slavnov-Taylor identities (STIs) $[95,96]$ satisfied by the corresponding vertices. Since the fully dressed vertices enter in the diagrammatic expansion of the gluon $\mathrm{SDE}$, their massless poles end up triggering the Schwinger mechanism, enabling a completely dynamical generation of an effective gluon mass [64,90-93].

It is clearly important to further scrutinize the dynamical picture described above, and identify certain characteristic properties that would corroborate its validity and discriminate it from alternative dynamical scenarios. In the present work we explore a distinctive signal of the non-Abelian Schwinger mechanism, which is intimately connected with the three-gluon vertex, and has the advantage of being reliably calculable by means of well-established inputs, such as two- and three-point correlation functions obtained from large-volume lattice simulations.

The pivotal ideas underlying this study may be summarized as follows. The massless poles are longitudinally coupled, and therefore drop out from "on-shell" observables $[49,88,89,97,98]$, or from the transversely projected vertices employed in lattice simulations [85,99-103], where only the pole-free part of the corresponding vertex survives. Nonetheless, the imprint of the poles is invariably encoded into the pole-free part, as may be seen by considering the Ward identity (WI) that this latter part satisfies, namely the limit of the STI as the gluon momentum in the channel of the pole is taken to zero. ${ }^{1}$

Since the poles contribute nontrivially to the STIs, the corresponding WI involves the standard building blocks (e.g., propagators) and a residual contribution with a nontrivial momentum dependence, which is directly related to the Schwinger mechanism. As a result, in that kinematic limit, the relevant form factor of the pole-free part of the vertex is displaced with respect to the case where the Schwinger mechanism is absent.

The above considerations become particularly relevant in the case of the three-gluon vertex, because the form factor of its pole-free part has been evaluated rather accurately in recent lattice simulations [104-106]. As a result, the displacement originating from the onset of the Schwinger mechanism, to be denoted by $\mathbb{C}\left(r^{2}\right)$, may be calculated by appropriately combining this form factor with all other constituents that enter into the WI of the three-gluon vertex; all of them are available from lattice simulations, with the exception of a particular partial derivative, denoted by $\mathcal{W}\left(r^{2}\right)$, related to the ghost-gluon kernel that appears in the STI $[107,108]$.

The importance of the calculation put forth above becomes particularly transparent when an additional theoretical ingredient is taken into account. Specifically, as will

\footnotetext{
${ }^{1}$ The standard Takahashi identity of QED, $q^{\mu} \Gamma_{\mu}(q, p$, $p+q)=S^{-1}(p+q)-S^{-1}(p)$, is an Abelian STI; the corresponding WI, $\Gamma_{\mu}(0, p,-p)=\partial S^{-1}(p) / \partial p^{\mu}$, is obtained from it by expanding around $q=0$.
}

become clear in the main body of the article, $\mathbb{C}\left(r^{2}\right)$ coincides exactly with the wave function amplitude of the massless bound state poles associated with the threegluon vertex [90-93]. Thus, the form of $\mathbb{C}\left(r^{2}\right)$ is determined from an entirely different procedure, namely as the solution of the BSE mentioned earlier. This solution, in turn, serves as a benchmark of our analysis, in the sense that signals emerging from the WI treatment are expected to be qualitatively compatible with the $\mathbb{C}\left(r^{2}\right)$ obtained from the BSE [90-93].

Our numerical analysis reveals that the $\mathbb{C}\left(r^{2}\right)$ constructed by putting together all the ingredients of the WI deviates markedly from zero, showing an impressive resemblance to the results obtained from the corresponding BSE. On average, the signal obtained is $3.1 \sigma$ away from the null hypothesis value, $\mathbb{C}\left(r^{2}\right)=0$, which corresponds to the absence of the Schwinger mechanism. Moreover, for momenta $r \geq 2 \mathrm{GeV}$ the deviation of the signal from $\mathbb{C}\left(r^{2}\right)=0$ exceeds the $5 \sigma$, owing to a characteristic peak of $\mathbb{C}\left(r^{2}\right)$ in the vicinity of $2 \mathrm{GeV}$, and to the fact that the error bars assigned to the lattice points get reduced as one moves away from the deep infrared region.

Let us finally mention that the principal uncertainty associated with the WI determination originates from the computation of the function $\mathcal{W}\left(r^{2}\right)$, which is not available from lattice simulations, and has been approximated by a truncated version of the SDE of the ghost-gluon kernel. As was explained in [108], the simulation of this function on the lattice is theoretically conceivable, but practically rather cumbersome.

The article is organized as follows. In Sec. II we explain in an Abelian context how the presence of longitudinally coupled massless poles modifies the form of the WI satisfied by the pole-free part of a vertex. In Sec. III we derive the corresponding WI for the pole-free part of the three-gluon vertex, introducing the displacement function $\mathbb{C}\left(r^{2}\right)$. Then, in Sec. IV we express $\mathbb{C}\left(r^{2}\right)$ in terms of the three-gluon form factor, the gluon propagator and its derivative, the ghost dressing function, and the function $\mathcal{W}\left(r^{2}\right)$. Next, in Sec. V we present the BSE determination of $\mathbb{C}\left(r^{2}\right)$. In Sec. VI we use lattice inputs for the components of the WI in order to determine the form of $\mathbb{C}\left(r^{2}\right)$, and compare it to the corresponding result obtained from the BSE. In Sec. VII we discuss issues related to $\mathbb{C}\left(r^{2}\right)$ and its physical implications. Finally, in Sec. VIII we present our discussion and conclusions. In addition, certain topics have been relegated to three appendices: Appendix A contains technical details of the BSE treatment, in Appendix B we discuss the SDE-based determination of $\mathcal{W}\left(r^{2}\right)$, while in Appendix $\mathrm{C}$ we collect the fits employed in our numerical analysis. 


\section{WARD IDENTITIES IN THE PRESENCE OF MASSLESS POLES}

In this section we focus on the modifications induced to the form of the WIs when the vertices involved contain longitudinally coupled massless poles, which is one of the trademarks of the Schwinger mechanism at the level of the vertices.

In general, the derivation of the WI from the corresponding Takahashi identity, or, in general, from a given STI, involves a Taylor expansion around the contracting momentum [67]. In the case of a function of a single variable, $f\left((p+q)^{2}\right)$, such as a propagator, the Taylor expansion proceeds through the elementary formula $(q \rightarrow 0)$

$$
\begin{aligned}
f\left((p+q)^{2}\right) & =f\left(p^{2}\right)+q^{\alpha} \frac{\partial f\left(p^{2}\right)}{\partial p^{\alpha}}+\mathcal{O}\left(q^{2}\right) \\
& =f\left(p^{2}\right)+2(q \cdot p) \frac{\partial f\left(p^{2}\right)}{\partial p^{2}}+\mathcal{O}\left(q^{2}\right) .
\end{aligned}
$$

For a function $f(q, r, p)$, with $q+r+p=0$, such as a three-particle vertex or kernel, the Taylor expansion around $q=0$ (and $p=-r$ ) gives

$f(q, r, p)=f(0, r,-r)+q^{\alpha}\left[\frac{\partial f(q, r, p)}{\partial q^{\alpha}}\right]_{q=0}+\mathcal{O}\left(q^{2}\right)$.

Note that if the $f(q, r, p)=-f(q, p, r)$, as happens in the case of the term associated with the massless pole in the $q$ channel (see below), then $f(0, r,-r)=0$.

In order to fix the ideas, we employ a vertex with reduced tensorial structure, which obeys an Abelian STI. In particular, we consider one of the typical vertices of the background field method (BFM) [109-116], namely the $B(q) \bar{c}(r) c(p)$ vertex, where $B$ denotes the background gluon ${ }^{2}$ and $\bar{c}(c)$ the antighost (ghost) fields. Due to the residual invariance of the action under background gauge transformations, this vertex satisfies an Abelian STI that relates it to the inverse ghost propagator. Specifically, suppressing the gauge coupling $g$ and the color factor $f^{a b c}$, and denoting the remainder of the vertex by $\tilde{\Gamma}_{\alpha}(q, r, p)$, we have $[6,60]$

$$
q^{\alpha} \tilde{\Gamma}_{\alpha}(q, r, p)=D^{-1}\left(p^{2}\right)-D^{-1}\left(r^{2}\right),
$$

where the ghost propagator is given by $D^{a b}\left(q^{2}\right)=$ $i \delta^{a b} D\left(q^{2}\right)$. Note that, at tree level, $\tilde{\Gamma}_{0}^{\alpha}(q, r, p)=(r-p)^{\alpha}$.

At this point we will assume that the Schwinger mechanism is inactive, such that the form factors comprising $\tilde{\Gamma}_{\alpha}(q, r, p)$ do not contain poles. In that case, one may

\footnotetext{
${ }^{2}$ Within the BFM, the gauge field $A_{\alpha}^{a}$ is decomposed as $A_{\alpha}^{a}=B_{\alpha}^{a}+Q_{\alpha}^{a}$, where $B_{\alpha}^{a}$ is the background field and $Q_{\alpha}^{a}$ is the quantum (fluctuating) field.
}

carry out the Taylor expansion of both sides of Eq. (2.3) according to Eqs. (2.1) and (2.2). Specifically, the left-hand side (1.h.s) of (2.3) yields

$$
[1 . h . s]=q^{\alpha} \tilde{\Gamma}_{\alpha}(0, r,-r)+\mathcal{O}\left(q^{2}\right),
$$

while the right-hand side (r.h.s) is simply given by

$$
[\text { r.h.s }]=q^{\alpha} \frac{\partial D^{-1}\left(r^{2}\right)}{\partial r^{\alpha}}+\mathcal{O}\left(q^{2}\right)
$$

Then, equating the coefficients of the terms linear in $q^{\alpha}$ on both sides, one obtains the simple, QED-like relation [67]

$$
\tilde{\Gamma}_{\alpha}(0, r,-r)=\frac{\partial D^{-1}\left(r^{2}\right)}{\partial r^{\alpha}}
$$

Finally, at the level of the single form factor comprising $\tilde{\Gamma}_{\alpha}(0, r,-r)$, namely

$$
\tilde{\Gamma}_{\alpha}(0, r,-r)=\tilde{\mathcal{A}}\left(r^{2}\right) r_{\alpha},
$$

we obtain directly from Eq. (2.6) [67]

$$
\tilde{\mathcal{A}}\left(r^{2}\right)=2 \frac{\partial D^{-1}\left(r^{2}\right)}{\partial r^{2}} .
$$

Let us now turn the Schwinger mechanism on, and denote the resulting full vertex by $\widetilde{\mathrm{I}}_{\alpha}(q, r, p)$. The vertex $\widetilde{\mathrm{I}}_{\alpha}(q, r, p)$, diagrammatically represented in Fig. 1, is comprised by two distinct pieces,

$$
\widetilde{\mathrm{I}}_{\alpha}(q, r, p)=\tilde{\Gamma}_{\alpha}(q, r, p)+\tilde{V}_{\alpha}(q, r, p),
$$

where $\tilde{\Gamma}_{\alpha}(q, r, p)$ contains all pole-free contributions, while the pole term $\tilde{V}_{\alpha}(q, r, p)$ has the general form $[49,88,89,97,98]$,

$$
\tilde{V}_{\alpha}(q, r, p)=\frac{q_{\alpha}}{q^{2}} \tilde{C}(q, r, p) .
$$

We emphasize that the pole-free terms $\tilde{\Gamma}_{\alpha}(q, r, p)$ are different when the Schwinger mechanism is turned on or off. In particular, the infrared finiteness of the gluon propagator affects the behavior of all other Green's functions, due to its nontrivial interconnection with them imposed by the corresponding coupled SDEs. A typical qualitative example of the type of modifications that the emergence of a gluonic mass scale induces to one-loop contributions is the conversion of "unprotected" logarithms into "protected" ones, according to $\ln \left(q^{2} / \mu^{2}\right) \rightarrow$ $\ln \left[\left(q^{2}+m^{2}\right) / \mu^{2}\right][101,117]$. 


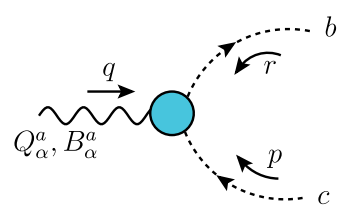

(a)

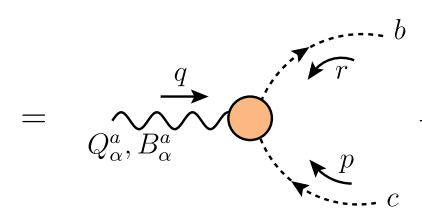

(b)

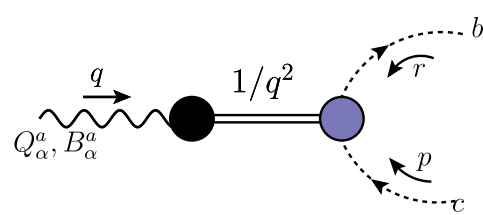

(c)

FIG. 1. (a) Compact diagrammatic representation of the vertices $\widetilde{I}_{\alpha}(q, r, p)$ ( $B$ gluon) and $\mathrm{I}_{\alpha}(q, r, p)(Q$ gluon); (b) the pole-free parts, $\tilde{\Gamma}_{\alpha}(q, r, p)$ and $\Gamma_{\alpha}(q, r, p)$; (c) the pole parts, $\tilde{V}_{\alpha}(q, r, p)$ and $V_{\alpha}(q, r, p)$. The black circle denotes the "transition amplitude," describing the mixing of the gluon with a massless excitation; its detailed diagrammatic content may be found in [91].

Evidently, combining Eqs. (2.9) and (2.10) we get

$$
q^{\alpha} \widetilde{\Gamma}_{\alpha}(q, r, p)=q^{\alpha} \tilde{\Gamma}_{\alpha}(q, r, p)+\tilde{C}(q, r, p)
$$

thus, the contraction by $q^{\alpha}$ cancels the massless pole in $q^{2}$.

We next assume that the Becchi-Rouet-Stora-Tyutin (BRST) symmetry $[118,119]$ of the theory remains intact as the Schwinger mechanism becomes operational. In this context, there are three important points that are worth emphasizing. First, a sharp distinction must be drawn between the standard BRST symmetry, understood throughout the present work, and the modified nonperturbative BRST symmetry exhibited by the refined GribovZwanziger action [68], as first demonstrated in [120]. Second, the nonperturbative quartet mechanism (see [121] and references therein) may be intrinsically related with the Schwinger mechanism, in the sense that it too relies on the dynamical formation of massless bound states (see additional comments in Sec. VIII). Third, motivated by arguments based mostly on the Kugo-Ojima formalism [122], a nonvanishing infrared-finite gluon propagator has often been associated with a soft breaking of the standard BRST symmetry (see, e.g., $[3,62]$ ); nonetheless, this conclusion depends on the precise mechanism responsible for this special behavior. In particular, the Schwinger mechanism, implemented via longitudinally coupled massless poles $[49,50,61,64,89,90,98,123]$, appears to be compatible with an unbroken BRST symmetry [67,124], at least at the level of the STIs satisfied by the elementary vertices. In particular, the STIs retain their standard form, but are now realized through the nontrivial participation of the massless pole terms.

Accordingly, the full $\widetilde{\mathrm{I}}_{\alpha}(q, r, p)$ satisfies, as before, precisely (2.3), namely

$$
q^{\alpha} \widetilde{\Gamma}_{\alpha}(q, r, p)=D^{-1}\left(p^{2}\right)-D^{-1}\left(r^{2}\right)
$$

where $D\left(q^{2}\right)$ is the ghost propagator in the presence of the Schwinger mechanism. For the same reasons described above for the case of $\tilde{\Gamma}_{\alpha}(q, r, p), D\left(q^{2}\right)$ also differs from the corresponding quantity when the Schwinger mechanism is not operational.
Then, using Eq. (2.11), we obtain for the pole-free part

$$
q^{\alpha} \tilde{\Gamma}_{\alpha}(q, r, p)=\left[D^{-1}\left(p^{2}\right)-D^{-1}\left(r^{2}\right)\right]-\tilde{C}(q, r, p) .
$$

The WI obeyed by $\tilde{\Gamma}_{\alpha}(q, r, p)$ may be derived again by means of a Taylor expansion, since, after the contraction by $q^{\alpha}$, all terms appearing in the STI of Eq. (2.13) contain no poles, as $q \rightarrow 0$. In particular,

$$
\begin{aligned}
q^{\alpha} \tilde{\Gamma}_{\alpha}(0, r,-r)= & \tilde{C}(0, r,-r) \\
& +q^{\alpha}\left\{\frac{\partial D^{-1}\left(r^{2}\right)}{\partial r^{\alpha}}-\left[\frac{\partial \tilde{C}(q, r, p)}{\partial q^{\alpha}}\right]_{q=0}\right\} \\
& +\mathcal{O}\left(q^{2}\right) .
\end{aligned}
$$

The comparison between Eqs. (2.14) and (2.5) reveals that the only zeroth-order contribution, namely $\tilde{C}(0, r,-r)$, must vanish,

$$
\tilde{C}(0, r,-r)=0
$$

Note that the result of Eq. (2.15) may be independently obtained from the property $\tilde{C}(q, r, p)=-\tilde{C}(q, p, r)$, which follows directly from the general ghost-antighost symmetry of the $B(q) \bar{c}(r) c(p)$ vertex.

Then, the matching of the terms linear in $q$ yields the WI

$$
\tilde{\Gamma}_{\alpha}(0, r,-r)=\frac{\partial D^{-1}\left(r^{2}\right)}{\partial r^{\alpha}}-\underbrace{\left[\frac{\partial \tilde{C}(q, r, p)}{\partial q^{\alpha}}\right]_{q=0}}_{\text {WI displacement }},
$$

which, when compared to that of Eq. (2.6), is "displaced" by the partial derivative of the form factor associated with the pole term.

In order to determine the displaced analog of Eq. (2.8), we set

$$
\left[\frac{\partial \tilde{C}(q, r, p)}{\partial q^{\alpha}}\right]_{q=0}=2 r_{\alpha} \tilde{\mathcal{C}}\left(r^{2}\right), \quad \tilde{\mathcal{C}}\left(r^{2}\right):=\left[\frac{\partial \tilde{C}(q, r, p)}{\partial p^{2}}\right]_{q=0},
$$

and obtain immediately from Eqs. (2.7) and (2.16) 


$$
\tilde{\mathcal{A}}\left(r^{2}\right)=2\left[\frac{\partial D^{-1}\left(r^{2}\right)}{\partial r^{2}}-\tilde{\mathcal{C}}\left(r^{2}\right)\right]
$$

Note that the displacement of the WI exemplified above becomes especially relevant within the framework that combines the pinch technique (PT) $[6,50,55,125]$ with the BFM, known as "PT-BFM scheme" [60,126]. In particular, the action of terms such as $\tilde{\mathcal{C}}\left(r^{2}\right)$ is instrumental for the evasion of a powerful nonperturbative cancellation that operates at the level of the gluon SDE [127], which would otherwise enforce the result $\Delta^{-1}(0)=0$. In fact, the contribution of the ghost loop to the nonvanishing $\Delta^{-1}(0)$, to be denoted by $\Delta_{\mathrm{gh}}^{-1}(0)$, is given by [67]

$$
\Delta_{\text {gh }}^{-1}(0) \sim \int d^{4} k k^{2} D^{2}\left(k^{2}\right) \tilde{\mathcal{C}}\left(k^{2}\right) .
$$

Let us finally point out that the displacement associated with the conventional ghost-gluon vertex $\mathrm{I}_{\alpha}(q, r, p)$ (see Fig. 1), to be denoted by $\mathcal{C}\left(r^{2}\right)$ [see Eq. (5.7)], is related to $\tilde{\mathcal{C}}\left(r^{2}\right)$ by the simple relation

$$
\mathcal{C}\left(r^{2}\right)=F(0) \tilde{\mathcal{C}}\left(r^{2}\right)
$$

where we have introduced the ghost dressing function, $F\left(q^{2}\right)$, related to the ghost propagator by $F\left(q^{2}\right)=$ $q^{2} D\left(q^{2}\right)$. The demonstration of Eq. (2.20) relies on the "background-quantum identity" that relates $\tilde{\mathrm{I}} \Gamma_{\alpha}(q, r, p)$ and $\mathrm{I} \Gamma_{\alpha}(q, r, p)[6,128]$; details will be presented elsewhere.

\section{THREE-GLUON VERTEX AND ITS WARD IDENTITY DISPLACEMENT}

In this section we consider the case of the three-gluon vertex in the conventional Landau gauge. If this vertex develops longitudinally coupled massless poles, its polefree part satisfies a displaced WI, whose derivation is the focal point of this section.

Before commencing, we introduce the gluon propagator, $\Delta_{\mu \nu}^{a b}(q)=-i \delta^{a b} \Delta_{\mu \nu}(q)$; in the Landau gauge that we employ in this work, it is given by the completely transverse form
$\Delta_{\mu \nu}(q)=\Delta\left(q^{2}\right) P_{\mu \nu}(q), \quad P_{\mu \nu}(q):=g_{\mu \nu}-q_{\mu} q_{\nu} / q^{2}$.

Furthermore, we define the two tensorial structures

$P_{\mu^{\prime}}^{\mu}(r) P_{\nu^{\prime}}^{\nu}(-r):=\mathcal{T}_{\mu^{\prime} \nu^{\prime}}^{\mu \nu}(r), \quad \lambda_{\mu \nu \alpha}(r):=2 r_{\alpha} P_{\mu \nu}(r)$,

and the tree-level three-gluon vertex, $\Gamma_{0}^{\alpha \mu \nu}(q, r, p)$, as

$\Gamma_{0}^{\alpha \mu \nu}(q, r, p)=(q-r)^{\nu} g^{\alpha \mu}+(r-p)^{\alpha} g^{\mu \nu}+(p-q)^{\mu} g^{\nu \alpha}$,

where the gauge coupling $g$ and the color factor $f^{a b c}$ were suppressed.

In order for the Schwinger mechanism to be activated, the full three-gluon vertex, to be denoted by $\mathrm{I}_{\alpha \mu \nu}^{a b c}(q, r, p)=$ $g f^{a b c} \mathrm{I \Gamma}_{\alpha \mu \nu}(q, r, p)$, is written as (see Fig. 2)

$$
\mathrm{I} \Gamma_{\alpha \mu \nu}(q, r, p)=\Gamma_{\alpha \mu \nu}(q, r, p)+V_{\alpha \mu \nu}(q, r, p),
$$

where $\Gamma_{\alpha \mu \nu}(q, r, p)$ is the pole-free component, while $V_{\alpha \mu \nu}(q, r, p)$ contains longitudinally coupled poles, i.e., it assumes the general form

$$
\begin{aligned}
V_{\alpha \mu \nu}(q, r, p)= & \left(\frac{q_{\alpha}}{q^{2}}\right) C_{\mu \nu}(q, r, p)+\left(\frac{r_{\mu}}{r^{2}}\right) A_{\alpha \nu}(q, r, p) \\
& +\left(\frac{p_{\nu}}{p^{2}}\right) B_{\alpha \mu}(q, r, p) .
\end{aligned}
$$

For the particular kinematic limit that we will eventually consider in the present work $(q \rightarrow 0)$, we only require the tensorial decomposition of the term $C_{\mu \nu}(q, r, p)$ in Eq. (3.5), given by

$$
\begin{aligned}
C_{\mu \nu}(q, r, p)= & C_{1} g_{\mu \nu}+C_{2} r_{\mu} r_{\nu}+C_{3} p_{\mu} p_{\nu} \\
& +C_{4} r_{\mu} p_{\nu}+C_{5} p_{\mu} r_{\nu},
\end{aligned}
$$

where $C_{i}:=C_{i}(q, r, p)$.

Due to its special form given by Eq. (3.5), $V_{\alpha \mu \nu}(q, r, p)$ satisfies the crucial condition

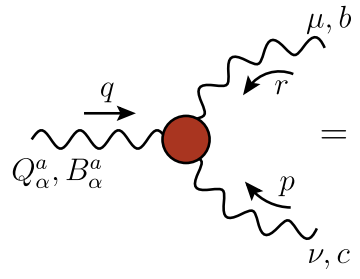

(a)

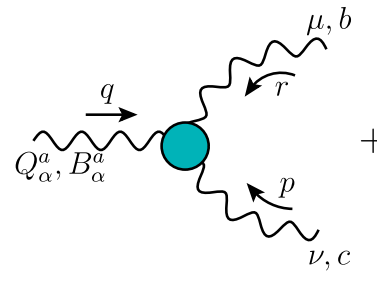

(b)

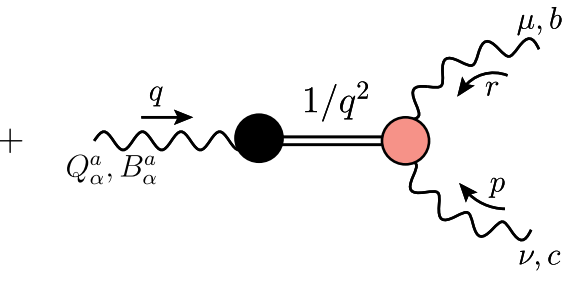

(c)

FIG. 2. (a) Compact diagrammatic representation of the vertices $\widetilde{I}_{\alpha \mu \nu}(q, r, p)\left(B\right.$ gluon) and $\operatorname{I\Gamma }_{\alpha \mu \nu}(q, r, p)(Q$ gluon); (b) the pole-free parts, $\tilde{\Gamma}_{\alpha \mu \nu}(q, r, p)$ and $\Gamma_{\alpha \mu \nu}(q, r, p)$; (c) the pole parts, $\tilde{V}_{\alpha \mu \nu}(q, r, p)$ and $V_{\alpha \mu \nu}(q, r, p)$. 


$$
P_{\alpha^{\prime}}^{\alpha}(q) P_{\mu^{\prime}}^{\mu}(r) P_{\nu^{\prime}}^{\nu}(p) V_{\alpha \mu \nu}(q, r, p)=0
$$

and, consequently, it drops out from the typical lattice observables involving the transversely projected threegluon vertex [see Eqs. (4.4) and (A2)].

The full vertex $\mathrm{I}_{\alpha \mu \nu}(q, r, p)$ satisfies the STI

$$
\begin{aligned}
q^{\alpha} I \Gamma_{\alpha \mu \nu}(q, r, p)= & F\left(q^{2}\right)\left[\Delta^{-1}\left(p^{2}\right) P_{\nu}^{\sigma}(p) H_{\sigma \mu}(p, q, r)\right. \\
& \left.-\Delta^{-1}\left(r^{2}\right) P_{\mu}^{\sigma}(r) H_{\sigma \nu}(r, q, p)\right] ;
\end{aligned}
$$

analogous expressions are obtained when contracting by $r^{\mu}$ or $p^{\nu}$. Note that the ghost-gluon kernel, $H_{\nu \mu}^{a b c}(q, p, r)=$ $-g f^{a b c} H_{\nu \mu}(q, p, r)$, defined in Fig. 12, enters in the STI nontrivially; for the nonperturbative structure of its relevant form factors, see [129]. In addition, we point out that the $H_{\sigma \mu}(p, q, r)$ and $H_{\sigma \nu}(r, q, p)$ also contain massless poles in the $r_{\mu}$ and $p_{\nu}$ channels, respectively, which are completely eliminated by the transverse projections in Eq. (3.12).

It is clear from Eqs. (3.4) and (3.6) that

$$
\begin{aligned}
& P_{\mu^{\prime}}^{\mu}(r) P_{\nu^{\prime}}^{\nu}(p)\left[q^{\alpha} \mathrm{I} \Gamma_{\alpha \mu \nu}(q, r, p)\right] \\
& \quad=P_{\mu^{\prime}}^{\mu}(r) P_{\nu^{\prime}}^{\nu}(p)\left[q^{\alpha} \Gamma_{\alpha \mu \nu}(q, r, p)+C_{\mu \nu}(q, r, p)\right],
\end{aligned}
$$

while, from the STI of Eq. (3.8)

$$
\begin{aligned}
& P_{\mu^{\prime}}^{\mu}(r) P_{\nu^{\prime}}^{\nu}(p)\left[q^{\alpha} \mathrm{I} \Gamma_{\alpha \mu \nu}(q, r, p)\right] \\
& \quad=P_{\mu^{\prime}}^{\mu}(r) P_{\nu^{\prime}}^{\nu}(p) F\left(q^{2}\right) R_{\nu \mu}(p, q, r),
\end{aligned}
$$

where

$R_{\nu \mu}(p, q, r):=\Delta^{-1}\left(p^{2}\right) H_{\nu \mu}(p, q, r)-\Delta^{-1}\left(r^{2}\right) H_{\mu \nu}(r, q, p)$.
Then, equating the right-hand sides of Eqs. (3.9) and (3.10) we obtain

$$
\begin{aligned}
q^{\alpha} & {\left[P_{\mu^{\prime}}^{\mu}(r) P_{\nu^{\prime}}^{\nu}(p) \Gamma_{\alpha \mu \nu}(q, r, p)\right] } \\
& =P_{\mu^{\prime}}^{\mu}(r) P_{\nu^{\prime}}^{\nu}(p)\left[F\left(q^{2}\right) R_{\nu \mu}(p, q, r)-C_{\mu \nu}(q, r, p)\right] .
\end{aligned}
$$

Due to the presence of the projectors $P_{\mu^{\prime}}^{\mu}(r) P_{\nu^{\prime}}^{\nu}(p)$, it is clear from Eq. (3.6) that only the terms $C_{1} g_{\mu \nu}$ and $C_{5} p_{\mu} r_{\nu}$ contribute to $C_{\mu \nu}(q, r, p)$. Note, however, that since $P_{\mu^{\prime}}^{\mu}(r) P_{\nu^{\prime}}^{\nu}(p) C_{5} p_{\mu} r_{\nu}=P_{\mu^{\prime}}^{\mu}(r) P_{\nu^{\prime}}^{\nu}(p) C_{5} q_{\mu} q_{\nu}$, this term is subleading, i.e., of order $\mathcal{O}\left(q^{2}\right)$, when the limit $q \rightarrow 0$ is taken.

We next proceed with the implementation of the limit $q \rightarrow 0$. In particular, as was done in the previous section, we carry out the Taylor expansion of both sides of Eq. (3.12) around $q=0$, and collect terms linear in $q$.

The computation of the 1.h.s. of Eq. (3.12) is immediate: using Eq. (3.2), we have

$$
[\text { l.h.s }]=q^{\alpha} \mathcal{T}_{\mu^{\prime} \nu^{\prime}}^{\mu \nu}(r) \Gamma_{\alpha \mu \nu}(0, r,-r)+\mathcal{O}\left(q^{2}\right) .
$$

The computation of the r.h.s. of Eq. (3.12) is considerably more complicated. We start by noticing that, to lowest order in $q$, only the term $C_{1}(q, r, p) g_{\mu \nu}$ survives. In addition, since it is clear from Eq. (3.11) that $R_{\nu \mu}(-r, 0, r)=0$, the vanishing of the zeroth order contribution imposes the condition

$$
C_{1}(0, r,-r)=0 \text {, }
$$

in exact analogy to Eq. (2.15).

Thus, the r.h.s. of Eq. (3.12) becomes

$$
[\text { r.h.s }]=q^{\alpha} \mathcal{T}_{\mu^{\prime} \nu^{\prime}}^{\mu \nu}(r) F(0)\left[\frac{\partial R_{\nu \mu}(p, q, r)}{\partial q^{\alpha}}\right]_{q=0}-q^{\alpha} P_{\mu^{\prime} \nu^{\prime}}(r)\left[\frac{\partial C_{1}(q, r, p)}{\partial q^{\alpha}}\right]_{q=0}+\mathcal{O}\left(q^{2}\right) .
$$

In order to compute the first partial derivative in Eq. (3.15), we exploit the fact that, in the Landau gauge, the ghost-gluon kernel may be cast in the form $[91,107]$

$$
H_{\nu \mu}(p, q, r)=\tilde{Z}_{1} g_{\nu \mu}+q^{\rho} K_{\nu \mu \rho}(p, q, r), \quad H_{\mu \nu}(r, q, p)=\tilde{Z}_{1} g_{\mu \nu}+q^{\rho} K_{\mu \nu \rho}(r, q, p),
$$

where the kernels $K$ do not contain poles as $q \rightarrow 0$. Moreover, $\tilde{Z}_{1}$ is the finite constant renormalizing $H_{\nu \mu}(p, q, r)$ in the "asymmetric" momentum subtraction (MOM) scheme, employed in the lattice simulation of [85,101]; its numerical value, estimated in [130], is $\tilde{Z}_{1} \approx 0.95$. 
Then, to lowest order in $q$,

$$
\begin{aligned}
& {\left[\frac{\partial H_{\nu \mu}(p, q, r)}{\partial q^{\alpha}}\right]_{q=0}=K_{\nu \mu \alpha}(-r, 0, r),} \\
& {\left[\frac{\partial H_{\mu \nu}(r, q, p)}{\partial q^{\alpha}}\right]_{q=0}=K_{\mu \nu \alpha}(r, 0,-r) .}
\end{aligned}
$$

Consider next the tensor decomposition of $K_{\mu \nu \alpha}(r, 0,-r)$ [107],

$$
K_{\mu \nu \alpha}(r, 0,-r)=K\left(r^{2}\right) g_{\mu \nu} r_{\alpha}+\cdots,
$$

where the ellipses denote terms proportional to $g_{\nu \alpha} r_{\mu}, g_{\mu \alpha} r_{\nu}$, and $r_{\alpha} r_{\mu} r_{\nu}$, which get annihilated by contraction with $\mathcal{T}_{\mu^{\prime} \nu^{\prime}}^{\mu \nu}(r)$. Clearly, $\mathcal{T}_{\mu^{\prime} \nu^{\prime}}^{\mu \nu}(r) K_{\nu \mu \alpha}(-r, 0, r)=$ $-\mathcal{T}_{\mu^{\prime} \nu^{\prime}}^{\mu \nu}(r) K_{\mu \nu \alpha}(r, 0,-r)$.

Then, it is straightforward to demonstrate that

$$
\begin{aligned}
& \mathcal{T}_{\mu^{\prime} \nu^{\prime}}^{\mu \nu}(r)\left[\frac{\partial R_{\nu \mu}(p, q, r)}{\partial q^{\alpha}}\right]_{q=0} \\
& \quad=\lambda_{\mu^{\prime} \nu^{\prime} \alpha}(r)\left\{\tilde{Z}_{1}\left[\Delta^{-1}\left(r^{2}\right)\right]^{\prime}-K\left(r^{2}\right) \Delta^{-1}\left(r^{2}\right)\right\},
\end{aligned}
$$

where the "prime" denotes differentiation with respect to $r^{2}$.

As for the second partial derivative in Eq. (3.15), applying the chain rule we have

$$
\left[\frac{\partial C_{1}(q, r, p)}{\partial q^{\alpha}}\right]_{q=0}=2 r_{\alpha} \mathbb{C}\left(r^{2}\right), \quad \mathbb{C}\left(r^{2}\right):=\left[\frac{\partial C_{1}(q, r, p)}{\partial p^{2}}\right]_{q=0},
$$

such that

$$
P_{\mu^{\prime} \nu^{\prime}}(r)\left[\frac{\partial C_{1}(q, r, p)}{\partial q^{\alpha}}\right]_{q=0}=\lambda_{\mu^{\prime} \nu^{\prime} \alpha}(r) \mathbb{C}\left(r^{2}\right)
$$

and, therefore, Eq. (3.15) becomes

$$
[\text { r.h.s }]=q^{\alpha} \lambda_{\mu^{\prime} \nu^{\prime} \alpha}(r)\left[F(0)\left\{\tilde{Z}_{1}\left[\Delta^{-1}\left(r^{2}\right)\right]^{\prime}-K\left(r^{2}\right) \Delta^{-1}\left(r^{2}\right)\right\}-\mathbb{C}\left(r^{2}\right)\right]+\mathcal{O}\left(q^{2}\right) .
$$

The final step is to equate the terms linear in $q$ that appear in Eqs. (3.13) and (3.22), to obtain the WI

$$
\mathcal{T}_{\mu^{\prime} \nu^{\prime}}^{\mu \nu}(r) \Gamma_{\alpha \mu \nu}(0, r,-r)=\lambda_{\mu^{\prime} \nu^{\prime} \alpha}(r)\left[F(0)\left\{\tilde{Z}_{1}\left[\Delta^{-1}\left(r^{2}\right)\right]^{\prime}-K\left(r^{2}\right) \Delta^{-1}\left(r^{2}\right)\right\}-\mathbb{C}\left(r^{2}\right)\right]
$$

Thus, the inclusion of the term $V_{\alpha \mu \nu}(q, r, p)$ in the vertex of Eq. (3.4) leads ultimately to the displacement of the WI satisfied by the pole-free part $\Gamma_{\alpha \mu \nu}(q, r, p)$, by an amount given by the special function $\mathbb{C}\left(r^{2}\right)$. Evidently, if $\mathbb{C}\left(r^{2}\right)=0$ one recovers the WI in the absence of the Schwinger mechanism.

We end this section with some remarks related to the PT-BFM scheme. Note that if the gauge field carrying the momentum $q$ is a background gluon instead of a quantum one (see Fig. 2), then the corresponding three-gluon vertex, $\tilde{\Gamma}_{\alpha \mu \nu}(q, r, p)$, satisfies a simplified version of the WI in Eq. (3.23), where $F \rightarrow 1, \tilde{Z}_{1} \rightarrow 1$, and $K\left(r^{2}\right) \rightarrow 0$, i.e.,

$\mathcal{T}_{\mu^{\prime} \nu^{\prime}}^{\mu \nu}(r) \tilde{\Gamma}_{\alpha \mu \nu}(0, r,-r)=\lambda_{\mu^{\prime} \nu^{\prime} \alpha}(r)\left[\left[\Delta^{-1}\left(r^{2}\right)\right]^{\prime}-\tilde{\mathbb{C}}\left(r^{2}\right)\right]$.

As has been demonstrated in [67], the contribution of the gluon loops to the nonvanishing $\Delta^{-1}(0)$, to be denoted by $\Delta_{\mathrm{gl}}^{-1}(0)$, is controlled by $\tilde{\mathbb{C}}\left(r^{2}\right)$,
$\Delta_{\mathrm{gl}}^{-1}(0) \sim \int d^{4} k k^{2} \Delta^{2}\left(k^{2}\right)\left[1-6 \pi \alpha_{s} C_{\mathrm{A}} Y\left(k^{2}\right)\right] \tilde{\mathbb{C}}\left(k^{2}\right)$,

where $\alpha_{s}:=g^{2} / 4 \pi, C_{\mathrm{A}}$ is the Casimir eigenvalue of the adjoint representation [ $N$ for $S U(N)$ ], and $Y\left(k^{2}\right)$ represents a particular one-loop correction (see, e.g., Fig. 3 in [67]). Evidently, Eq. (3.25) is the exact analog of Eq. (2.19). The total mass, identified with $\Delta^{-1}(0)$, is obtained by summing up Eqs. (3.25) and (2.19).

Finally, the relation between $\tilde{\mathbb{C}}\left(r^{2}\right)$ and $\mathbb{C}\left(r^{2}\right)$ is given by

$$
\mathbb{C}\left(r^{2}\right)=F(0) \tilde{\mathbb{C}}\left(r^{2}\right),
$$

in exact analogy to Eq. (2.20).

\section{DISPLACEMENT FUNCTION IN TERMS OF LATTICE QUANTITIES}

In this section we establish a crucial connection between the 1.h.s. of Eq. (3.23) and the results of recent lattice simulations. This, in turn, will allow us to relate the 
characteristic ingredient of the Schwinger mechanism, namely $\mathbb{C}\left(r^{2}\right)$, to quantities obtained directly from lattice QCD. The advantage of such a connection is that the lattice is intrinsically "blind" to particular field theoretic constructs (such as the Schwinger mechanism), furnishing results obtained through the model-independent functional averaging over gauge-field configurations.

We start our analysis by considering the pole-free part $\Gamma_{\alpha \mu \nu}(q, r, p)$ of the three-gluon vertex, in the kinematic limit of interest, $q \rightarrow 0$. Given that only a single momentum $(r)$ is available, the general tensorial decomposition of $\Gamma_{\alpha \mu \nu}(0, r,-r)$ is given by

$$
\begin{aligned}
\Gamma_{\alpha \mu \nu}(0, r,-r)= & 2 \mathcal{A}_{1}\left(r^{2}\right) r_{\alpha} g_{\mu \nu}+\mathcal{A}_{2}\left(r^{2}\right) \\
& \times\left(r_{\mu} g_{\nu \alpha}+r_{\nu} g_{\mu \alpha}\right)+\mathcal{A}_{3}\left(r^{2}\right) r_{\alpha} r_{\mu} r_{\nu},
\end{aligned}
$$

where the form factors $\mathcal{A}_{i}\left(r^{2}\right)$ may diverge at most logarithmically as $r \rightarrow 0$, but do not contain stronger singularities. At tree level, we have that

$$
\Gamma_{0}^{\alpha \mu \nu}(0, r,-r)=2 r^{\alpha} g^{\mu \nu}-\left(r^{\mu} g^{\nu \alpha}+r^{\nu} g^{\mu \alpha}\right),
$$

corresponding to $\mathcal{A}_{1}^{(0)}\left(r^{2}\right)=1, \quad \mathcal{A}_{2}^{(0)}\left(r^{2}\right)=-1, \quad$ and $\mathcal{A}_{3}^{(0)}\left(r^{2}\right)=0$.

It is then elementary to derive from Eq. (4.1) that

$$
\mathcal{T}_{\mu^{\prime} \nu^{\prime}}^{\mu \nu}(r) \Gamma_{\alpha \mu \nu}(0, r,-r)=\mathcal{A}_{1}\left(r^{2}\right) \lambda_{\mu^{\prime} \nu^{\prime} \alpha}(r)
$$

We next establish a connection between the form factor $\mathcal{A}_{1}\left(r^{2}\right)$ and the projection of the three-gluon vertex studied in the lattice simulations of $[85,99,100,102,103,106,131-136]$. Specifically, after appropriate amputation of the external legs, the lattice quantity $L_{s g}\left(r^{2}\right)$ is given by

$$
L_{s g}\left(r^{2}\right)=\left.\frac{\Gamma_{0}^{\alpha \mu \nu}(q, r, p) P_{\alpha \alpha^{\prime}}(q) P_{\mu \mu^{\prime}}(r) P_{\nu \nu^{\prime}}(p) I \Gamma^{\alpha^{\prime} \mu^{\prime} \nu^{\prime}}(q, r, p)}{\Gamma_{0}^{\alpha \mu \nu}(q, r, p) P_{\alpha \alpha^{\prime}}(q) P_{\mu \mu^{\prime}}(r) P_{\nu \nu^{\prime}}(p) \Gamma_{0}^{\alpha^{\prime} \mu^{\prime} \nu^{\prime}}(q, r, p)}\right|_{\substack{q \rightarrow 0 \\ p \rightarrow-r}}
$$

Now, by virtue of Eq. (3.7), it is clear that the term $V^{\alpha^{\prime} \mu^{\prime} \nu^{\prime}}(q, r, p)$ associated with the poles drops out from Eq. (4.4) in its entirety, amounting effectively to the replacement $\mathrm{I}^{\alpha^{\prime} \mu^{\prime} \nu^{\prime}}(q, r, p) \rightarrow \Gamma^{\alpha^{\prime} \mu^{\prime} \nu^{\prime}}(q, r, p)$.

Then, the numerator, $\mathcal{N}$, and denominator, $\mathcal{D}$, of the fraction on the r.h.s. of Eq. (4.4), after employing Eqs. (4.1) and (4.2), become

$$
\begin{aligned}
\mathcal{N} & =4(d-1)\left[r^{2}-(r \cdot q)^{2} / q^{2}\right] \mathcal{A}_{1}\left(r^{2}\right), \\
\mathcal{D} & =4(d-1)\left[r^{2}-(r \cdot q)^{2} / q^{2}\right] .
\end{aligned}
$$

Evidently, the path-dependent contribution contained in the square bracket drops out when forming the ratio $\mathcal{N} / \mathcal{D}$, and Eq. (4.4) yields the important relation

$$
L_{s g}\left(r^{2}\right)=\mathcal{A}_{1}\left(r^{2}\right) .
$$

Combining Eqs. (4.3) and (4.6), we get

$$
\mathcal{T}_{\mu^{\prime} \nu^{\prime}}^{\mu \nu}(r) \Gamma_{\alpha \mu \nu}(0, r,-r)=L_{s g}\left(r^{2}\right) \lambda_{\mu^{\prime} \nu^{\prime} \alpha}(r) .
$$

At this point, after substitution of Eq. (4.7) into Eq. (3.23), we arrive at

$\mathbb{C}\left(r^{2}\right)=F(0)\left\{\tilde{Z}_{1}\left[\Delta^{-1}\left(r^{2}\right)\right]^{\prime}-K\left(r^{2}\right) \Delta^{-1}\left(r^{2}\right)\right\}-L_{s g}\left(r^{2}\right)$.

The final step consists in passing the result of Eq. (4.8) from Minkowski to Euclidean space, following the standard conversion rules. Specifically, we set $r^{2}=-r_{\mathrm{E}}^{20}$, with $r_{\mathrm{E}}^{2}>0$ the positive square of an Euclidean four-vector, and use

$$
\begin{array}{ll}
\Delta_{\mathrm{E}}\left(r_{\mathrm{E}}^{2}\right)=-\Delta\left(-r_{\mathrm{E}}^{2}\right), & F_{\mathrm{E}}\left(r_{\mathrm{E}}^{2}\right)=F\left(-r_{\mathrm{E}}^{2}\right), \\
L_{s g}^{\mathrm{E}}\left(r_{\mathrm{E}}^{2}\right)=L_{s g}\left(-r_{\mathrm{E}}^{2}\right), & \mathbb{C}_{\mathrm{E}}\left(r_{\mathrm{E}}^{2}\right)=-\mathbb{C}\left(-r_{\mathrm{E}}^{2}\right) .
\end{array}
$$

In what follows we suppress the indices "E" to avoid notational clutter.

Then, Eq. (4.8) is converted to

$$
\mathbb{C}\left(r^{2}\right)=L_{s g}\left(r^{2}\right)+F(0)\left\{K\left(r^{2}\right) \Delta^{-1}\left(r^{2}\right)-\tilde{Z}_{1}\left[\Delta^{-1}\left(r^{2}\right)\right]^{\prime}\right\},
$$

which is one of the central results of this article.

Finally, it is convenient to introduce the dimensionless function $\mathcal{W}\left(r^{2}\right)$, defined as

$$
K\left(r^{2}\right)=-\frac{\mathcal{W}\left(r^{2}\right)}{r^{2}}
$$

thus casting Eq. (4.12) into the form

$\mathbb{C}\left(r^{2}\right)=L_{s g}\left(r^{2}\right)-F(0)\left\{\frac{\mathcal{W}\left(r^{2}\right)}{r^{2}} \Delta^{-1}\left(r^{2}\right)+\tilde{Z}_{1}\left[\Delta^{-1}\left(r^{2}\right)\right]^{\prime}\right\}$, 
which will be employed in the numerical evaluation given in Sec. VI.

\section{DYNAMICAL DETERMINATION OF THE DISPLACEMENT FUNCTION}

In this section we elaborate on the determination of $\mathbb{C}\left(r^{2}\right)$ from the BSEs that describe the dynamical formation of massless colored bound states; the analysis is based on the derivations given in [90,93], adapted to the present context. Note that this procedure determines also the analog of $\tilde{\mathcal{C}}\left(r^{2}\right)$, introduced in Sec. II, for the case of the conventional ghost-gluon vertex, to be denoted by $\mathcal{C}\left(r^{2}\right)$.

The starting point of this study is the BS version of the SDEs that govern the momentum evolution of the threegluon vertex, $\mathrm{I}_{\alpha \mu \nu}(q, r, p)$, and of the conventional ghostgluon vertex, $\mathrm{I} \Gamma_{\alpha}(q, r, p)$, shown in Fig. 3. In particular, we replace (inside the loops) the tree-level vertices (with incoming momentum $q$ ) by their fully dressed counterparts, modifying the corresponding multiparticle kernels $\mathcal{K}_{i j}$ accordingly, to avoid overcounting (see e.g., Fig. 7 of [90]). The main advantage of this conversion is that various vertex renormalization constants, which otherwise would appear explicitly multiplying the corresponding diagrams, are naturally absorbed by the additional dressed vertices. Note that, in order to simplify the pertinent set of SDEs, we omit from our analysis the fully dressed four-gluon vertices (with incoming momentum $q$ ), whose impact is expected to be subleading $[12,137]$.

In what follows, we will introduce a longitudinally coupled massless pole also in the ghost-gluon vertex $\mathrm{I} \Gamma_{\alpha}(q, r, p)$, casting it into a form analogous to Eq. (2.9), and diagrammatically represented in Fig. 1, where the incoming gluon is $Q_{\alpha}^{a}$. In particular, we set

$$
\mathrm{I} \Gamma_{\alpha}(q, r, p)=\Gamma_{\alpha}(q, r, p)+V_{\alpha}(q, r, p)
$$

where $\Gamma_{\alpha}(r, p, q)$ denotes the pole-free component, while

$$
V_{\alpha}(q, r, p)=\frac{q_{\alpha}}{q^{2}} C(q, r, p),
$$

describes the pole multiplied by the associated form factor.

Then, the BSEs of Fig. 3 may be written schematically as

$$
\begin{aligned}
\mathrm{I}^{\alpha \mu \nu}(q, r, p)= & \Gamma_{0}^{\alpha \mu \nu}(q, r, p)-\frac{i g^{2} C_{\mathrm{A}}}{2} \int_{k} \mathrm{I} \Gamma^{\alpha \beta \gamma}(q, k,-s) \Delta_{\beta \rho}(k) \Delta_{\gamma \sigma}(s) \mathcal{K}_{11}^{\mu \nu \sigma \rho}(r, p, s,-k) \\
& +i g^{2} C_{\mathrm{A}} \int_{k} \mathrm{I}^{\alpha}(q, k,-s) D\left(k^{2}\right) D\left(s^{2}\right) \mathcal{K}_{12}^{\mu \nu}(r, p, s,-k), \\
\mathrm{I}^{\alpha}(q, r, p)= & \Gamma_{0}^{\alpha}(q, r, p)-\frac{i g^{2} C_{\mathrm{A}}}{2} \int_{k} \mathrm{I} \Gamma^{\alpha \beta \gamma}(q, k,-s) \Delta_{\beta \rho}(k) \Delta_{\gamma \sigma}(s) \mathcal{K}_{21}^{\sigma \rho}(r, p, s,-k) \\
& -\frac{i g^{2} C_{\mathrm{A}}}{2} \int_{k} \mathrm{I}^{\alpha}(q, k,-s) D\left(k^{2}\right) D\left(s^{2}\right) \mathcal{K}_{22}(r, p, s,-k),
\end{aligned}
$$

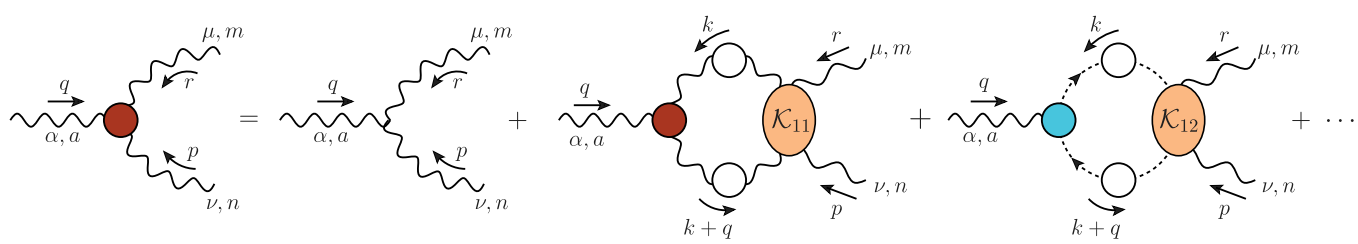

(a)

(b)

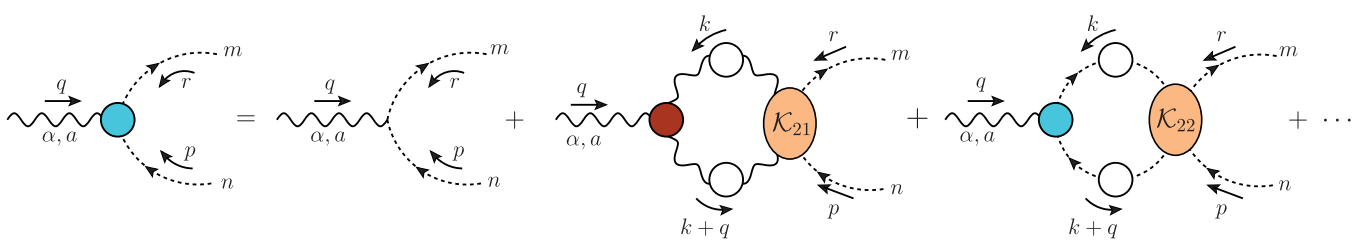

(c)

(d)

FIG. 3. The coupled system of inhomogeneous BSEs for the three-gluon and ghost-gluon vertices, $\mathrm{I} \Gamma^{\alpha \mu \nu}(q, r, p)$ and $\mathrm{I} \Gamma^{\alpha}(q, r, p)$, respectively. Circles represent full propagators or vertices, while the orange ellipses correspond to four-point scattering kernels, $\mathcal{K}_{i j}$. The omitted diagrams contain five-point scattering kernels, associated with fully dressed four-gluon vertices with incoming momentum $q$, which are neglected in our truncation scheme. 
where $\Gamma_{0}^{\alpha}(q, r, p)=r^{\alpha}$ is the tree-level ghost-gluon vertex, $s:=k+q$, and we introduce the notation

$$
\int_{k}:=\frac{1}{(2 \pi)^{4}} \int d^{4} k
$$

for the integral measure. In Eq. (5.4), the use of a symmetry-preserving regularization scheme is implicitly assumed.

Next, we decompose the vertices in Eq. (5.3) according to Eqs. (3.4) and (5.1). Given that $\mathrm{I} \Gamma_{\alpha \mu \nu}(q, r, p)$ contains poles in all channels, $q_{\alpha}, r_{\mu}$, and $p_{\nu}$, we isolate the pole in $q_{\alpha}$ by contracting the first line of Eq. (5.3) with $P^{\mu \delta}(r) P_{\delta}^{\nu}(p)$. Then, using Eqs. (3.5) and (3.6) we find that

$$
\begin{aligned}
P^{\mu \delta} & (r) P_{\delta}^{\nu}(p) V_{\alpha \mu \nu}(q, r, p) \\
\quad & =\frac{q_{\alpha}}{q^{2}} P^{\mu \delta}(r) P_{\delta}^{\nu}(p)\left[g_{\mu \nu} C_{1}(q, r, p)+q_{\mu} q_{\nu} C_{5}(q, r, p)\right],
\end{aligned}
$$

so that the only pole terms on the 1.h.s. of Eq. (5.3) are those containing $C_{1}$ and $C_{5}$. In addition, due to the transversality of the Landau gauge gluon propagator, Eq. (5.5) (with $q \rightarrow q, r \rightarrow k$, and $p \rightarrow-s$ ) can be used inside the integral of diagram (a); again, only $C_{1}(q, k,-s)$ and $C_{5}(q, k,-s)$ survive. Exactly the same situation is reproduced inside diagram (c).

The following step is to multiply Eq. (5.3) by $q^{2}$ and expand around $q=0$. In doing so, we recall Eqs. (3.14) and (3.20), and the analogous relations for $C(q, r, p)$, namely ${ }^{3}$

$$
C(0, r,-r)=0
$$

and

$$
\left[\frac{\partial C(q, r, p)}{\partial q^{\alpha}}\right]_{q=0}=2 r_{\alpha} \mathcal{C}\left(r^{2}\right), \quad \mathcal{C}\left(r^{2}\right):=\left[\frac{\partial C(q, r, p)}{\partial p^{2}}\right]_{q=0} .
$$

Then, as $q \rightarrow 0$, the term proportional to $C_{5}$ in Eq. (5.5) is of higher order in $q$ and drops out.

Consequently, we obtain a set of homogeneous equations involving only $\mathbb{C}\left(r^{2}\right)$ and $\mathcal{C}\left(r^{2}\right)$. Specifically, we find

$$
\begin{aligned}
3(q \cdot r) \mathbb{C}\left(r^{2}\right)= & -\frac{i g^{2} C_{\mathrm{A}}}{2} \int_{k}(q \cdot k) \mathbb{C}\left(k^{2}\right) \Delta^{2}\left(k^{2}\right) P_{\rho \sigma}(k) P_{\mu \nu}(r) \mathcal{K}_{11}^{\mu \nu \sigma \rho}(r,-r, k,-k) \\
& +i g^{2} C_{\mathrm{A}} \int_{k}(q \cdot k) \mathcal{C}\left(k^{2}\right) D^{2}\left(k^{2}\right) P_{\mu \nu}(r) \mathcal{K}_{12}^{\mu \nu}(r,-r, k,-k) \\
(q \cdot r) \mathcal{C}\left(r^{2}\right)= & -\frac{i g^{2} C_{\mathrm{A}}}{2} \int_{k}(q \cdot k) \mathbb{C}\left(k^{2}\right) \Delta^{2}\left(k^{2}\right) P_{\sigma \rho}(k) \mathcal{K}_{21}^{\sigma \rho}(r,-r, k,-k) \\
& -\frac{i g^{2} C_{\mathrm{A}}}{2} \int_{k}(q \cdot k) \mathcal{C}\left(k^{2}\right) D^{2}\left(k^{2}\right) \mathcal{K}_{22}(r,-r, k,-k)
\end{aligned}
$$

where we have used $P_{\mu}^{\mu}(r)=3$. Then, the remaining common factor of $q$ can be eliminated straightforwardly, by making use of the basic formula

$$
\int_{k}(q \cdot k) F(k, r)=\frac{(q \cdot r)}{r^{2}} \int_{k}(r \cdot k) F(k, r) .
$$

Finally, we approximate the four-point scattering kernels $\mathcal{K}_{i j}$ by their one-particle exchange diagrams (see e.g., Figs. 4 and 5 of [93]), thus reducing the BSEs governing $\mathbb{C}\left(r^{2}\right)$ and $\mathcal{C}\left(r^{2}\right)$ to the form shown in Fig. 4; the corresponding algebraic expressions are given in Eq. (A4).

\footnotetext{
${ }^{3}$ Equation (5.6) can be proved from an STI for the ghost-gluon vertex, in analogy to the proof leading to Eq. (2.15) using the Abelian STI of Eq. (2.11). The full derivation will be given elsewhere.
}

We observe that the system of integral equations reached in Eq. (5.8) is the (approximate) BSE that governs the formation of massless colored bound states $\left(q^{2}=0\right)$, as announced. ${ }^{4}$ Thus, the function $\mathbb{C}\left(r^{2}\right)$, connected with the displacement of the WI in Eq. (4.12), emerges naturally as the wave function associated with the pole formation of a colored two-gluon bound state.

We point out that, in addition to the lattice propagators given in Appendix C, the numerical evaluation of the BSEs requires information on various form factors of the polefree vertices $\Gamma_{\alpha \mu \nu}(q, r, p)$ and $\Gamma_{\alpha}(q, r, p)$; for details, see Appendix A.

\footnotetext{
${ }^{4}$ Note that the BSE derived as $q \rightarrow 0$ is identical to the one obtained as $q^{2} \rightarrow 0$; however, the former derivation is operationally simpler.
} 


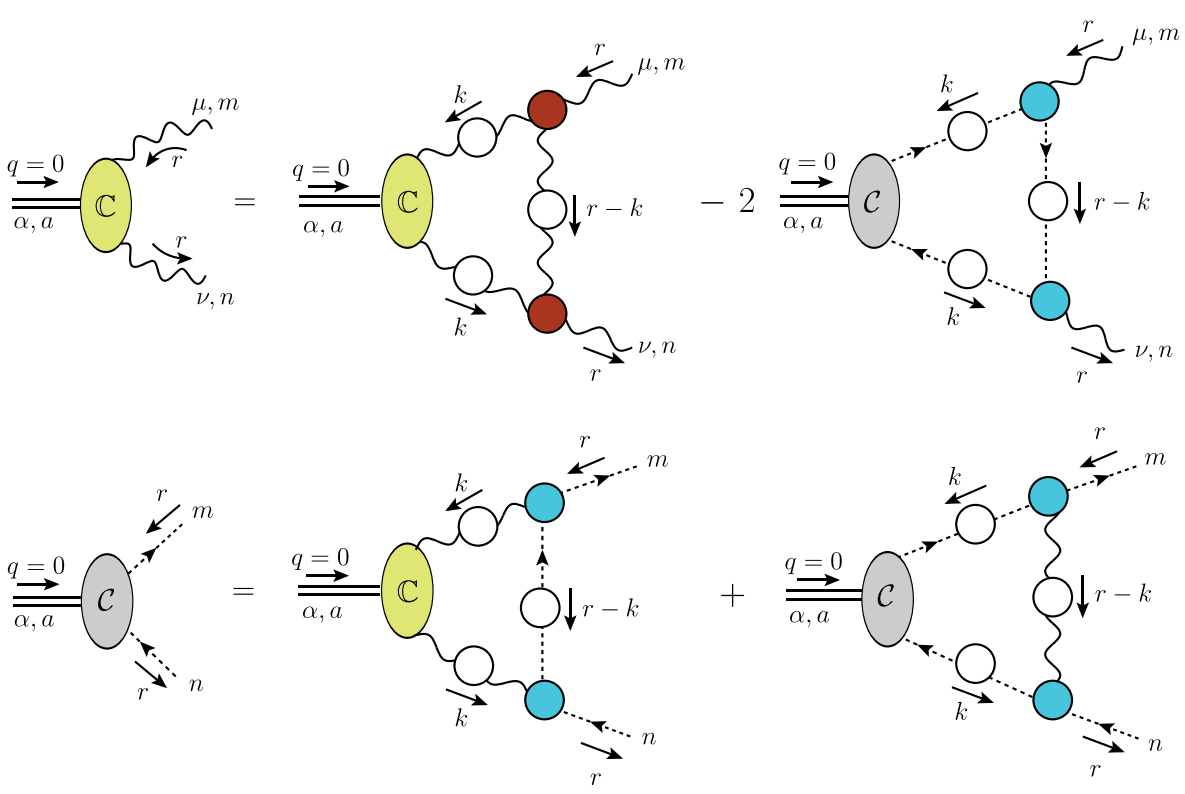

FIG. 4. The coupled system of BSEs governing the evolution of $\mathbb{C}\left(r^{2}\right)$ and $\mathcal{C}\left(r^{2}\right)$.

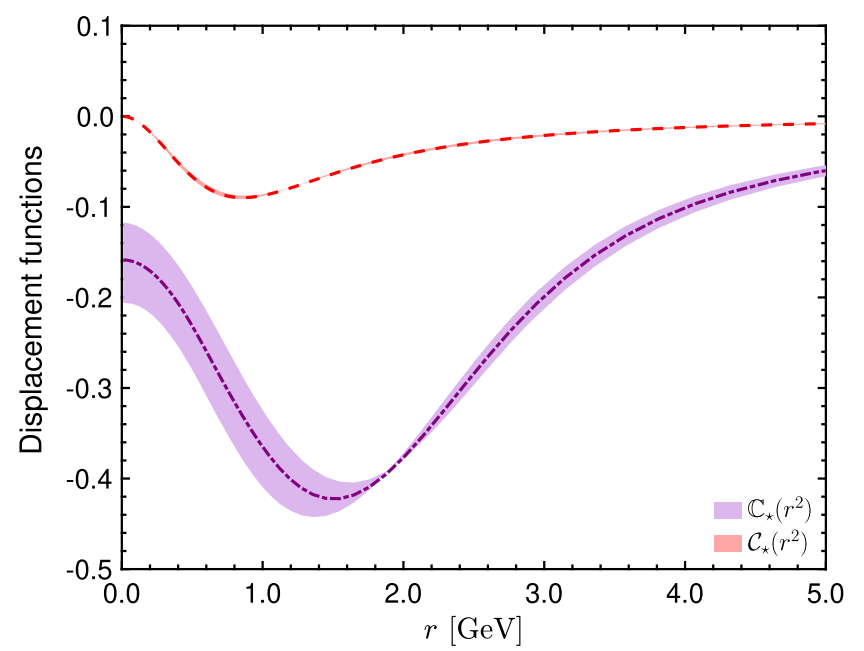

FIG. 5. The solutions for $\mathbb{C}_{\star}\left(r^{2}\right)$ (purple dot-dashed) and $\mathcal{C}_{\star}\left(r^{2}\right)$ (red dashed) obtained from the coupled BSE system of Eq. (A4). The origin of the error bands is the propagation of the error associated with $L_{s g}\left(r^{2}\right)$, used to calibrate the three-gluon vertex inputs. The band of $\mathcal{C}_{\star}\left(r^{2}\right)$ turns out to be very thin and barely visible.

We emphasize that, due to the homogeneity and linearity of Eq. (A4), the overall scale of the solution is undetermined: the multiplication of a given solution by an arbitrary real constant yields another solution. For the purposes of the present work, this ambiguity was resolved by matching the BSE prediction for $\mathbb{C}\left(r^{2}\right)$ to the result obtained from the WI in the next section. The solutions found for $\mathbb{C}\left(r^{2}\right)$ and $\mathcal{C}\left(r^{2}\right)$ after the implementation of this scale-fixing procedure, denoted as $\mathbb{C}_{\star}\left(r^{2}\right)$ and $\mathcal{C}_{\star}\left(r^{2}\right)$, respectively, are shown in Fig. 5. Note that $\mathbb{C}_{\star}\left(r^{2}\right)$ is considerably larger in magnitude than $\mathcal{C}_{\star}\left(r^{2}\right)$, in agreement with the original study presented in [93].

\section{DISPLACEMENT FUNCTION FROM THE WARD IDENTITY}

We next determine the signal for $\mathbb{C}\left(r^{2}\right)$ that emerges from the corresponding WI, and discuss its statistical significance with respect to the null hypothesis, namely the case where $\mathbb{C}\left(r^{2}\right)$ would vanish identically.

To that end, we substitute on the r.h.s. of Eq. (4.12) appropriate expressions for all quantities appearing there. In particular, we employ physically motivated fits to lattice results for the gluon propagator, the ghost dressing function, and $L_{s g}\left(r^{2}\right)$, given in Appendix C. Instead, the function $\mathcal{W}\left(r^{2}\right)$ is computed from its own SDE, as described in Appendix B; the resulting $\mathcal{W}\left(r^{2}\right)$ is shown as the blue solid line and error band in the right panel of Fig. 13.

The outcome of this operation is clearly nonvanishing: the resulting $\mathbb{C}\left(r^{2}\right)$ is shown in the left panel of Fig. 6 as the blue continuous curve, which is distinctly separated from the null hypothesis case, indicated by the green dotted line. The blue band surrounding the central result indicates the errors assigned to $\mathbb{C}\left(r^{2}\right)$, through the propagation of the corresponding errors associated with the ingredients entering on the r.h.s. of Eq. (4.12).

In the same figure we plot the $\mathbb{C}_{\star}\left(r^{2}\right)$ of Fig. 5, in order to facilitate the direct comparison. We observe an excellent agreement in the overall shapes of $\mathbb{C}_{\star}\left(r^{2}\right)$ and $\mathbb{C}\left(r^{2}\right)$. Their main difference is the position and depth of the minimum: for $\mathbb{C}\left(r^{2}\right)$ we have $r_{\text {min }}=1.95_{-0.10}^{+0.07}$ and $\mathbb{C}\left(r_{\text {min }}^{2}\right)=-0.39 \pm$ 0.08 , while for $\mathbb{C}_{\star}\left(r^{2}\right)$ we find $r_{\min }=1.50_{-0.13}^{+0.12}$ and $\mathbb{C}_{\star}\left(r_{\text {min }}^{2}\right)=-0.42 \pm 0.02$. 

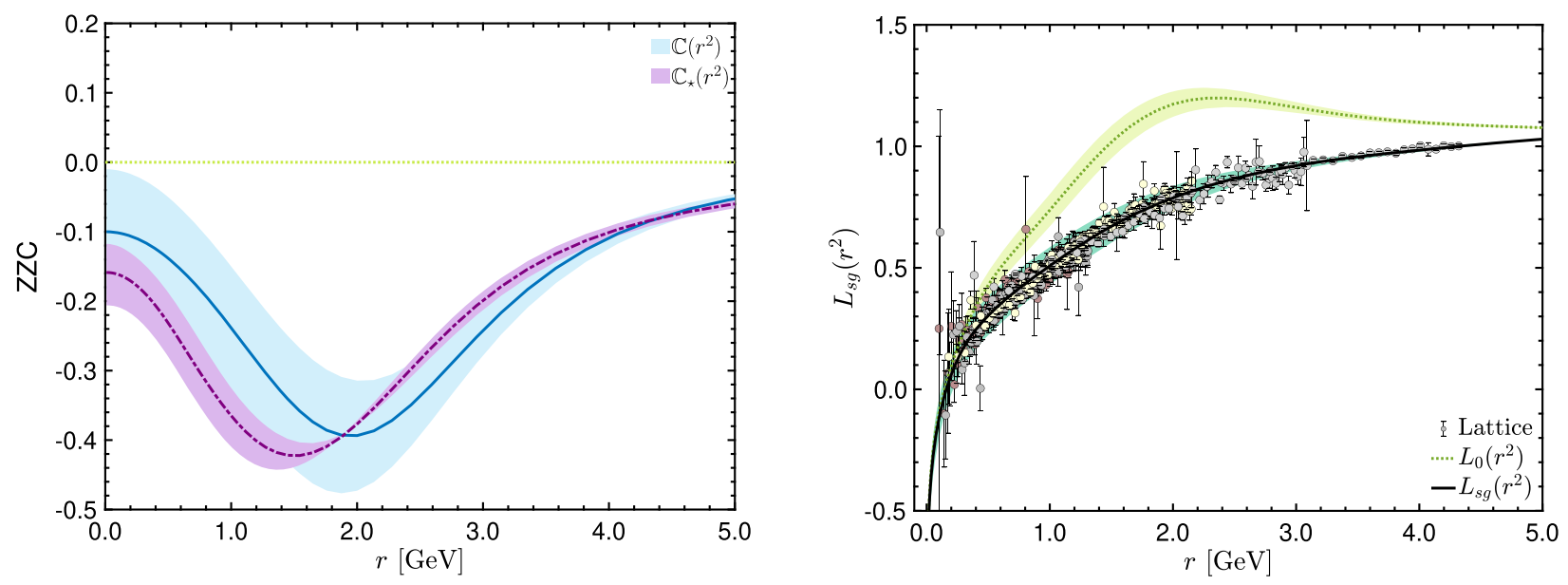

FIG. 6. Left: $\mathbb{C}\left(r^{2}\right)$ obtained from Eq. (4.12) (blue continuous curve) compared to the BSE prediction, $\mathbb{C}_{\star}\left(r^{2}\right)$, based on Eq. (A4) (purple dot-dashed). Right: lattice data of [106] for $L_{s g}\left(r^{2}\right)$ (points), compared to the fit of Eq. (C12) (black continuous), and the null hypothesis prediction, $L_{0}\left(r^{2}\right)$, of Eq. (6.1) (green dotted). The band around $L_{s g}\left(r^{2}\right)$ is delimited by the functions $L_{s g}^{ \pm}\left(r^{2}\right)$ of Eq. (C13).

In order to provide an estimate of the statistical significance of the above signal, we find it advantageous to recast our analysis in terms of the quantity $L_{s g}\left(r^{2}\right)$, thus capitalizing on the detailed error analysis applied to the lattice data of [106]. Specifically, from the WI of Eq. (4.12) we will determine the form that $L_{s g}\left(r^{2}\right)$ would have if the null hypothesis were true, and quantify its deviation from the actual lattice data.

Thus, setting $\mathbb{C}\left(r^{2}\right)=0$ into Eq. (4.12), we obtain the null hypothesis prediction for $L_{s g}\left(r^{2}\right)$, which we denote by $L_{0}\left(r^{2}\right)$, given by

$$
L_{0}\left(r^{2}\right)=F(0)\left\{\frac{\mathcal{W}\left(r^{2}\right) \Delta^{-1}\left(r^{2}\right)}{r^{2}}+\tilde{Z}_{1}\left[\Delta^{-1}\left(r^{2}\right)\right]^{\prime}\right\}
$$

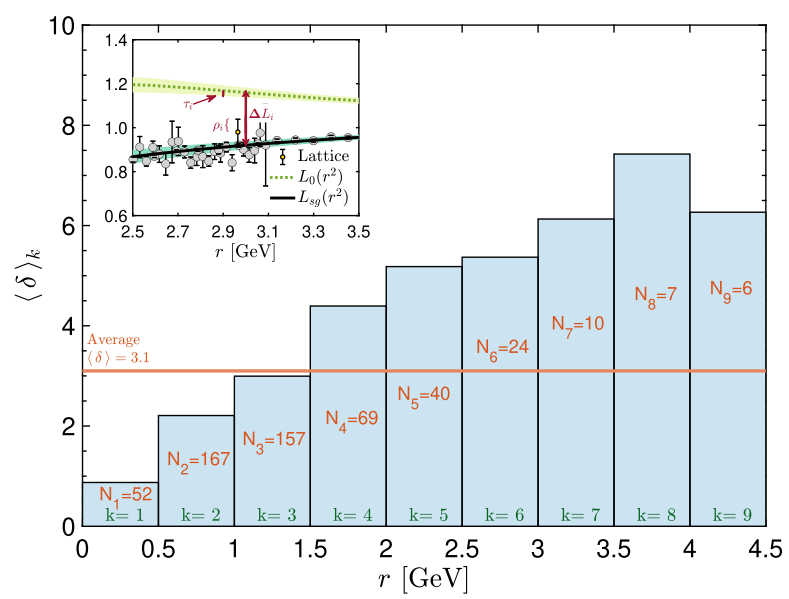

Substituting on the r.h.s. of Eq. (6.1) the same ingredients as before, we obtain the $L_{0}\left(r^{2}\right)$ shown as the green dotted line on the right panel of Fig. 6 . The green band enveloping $L_{0}\left(r^{2}\right)$ captures the error propagated from $\mathcal{W}\left(r^{2}\right)$; it is obtained by using as inputs into Eq. (6.1) the curves delimiting the blue band in the right panel of Fig. 13.

The results shown in Fig. 6 demonstrate that the statistical error of the lattice cannot account for the discrepancy between $L_{s g}\left(r^{2}\right)$ and $L_{0}\left(r^{2}\right)$; evidently, the null hypothesis is strongly disfavored.

In order to quantify the above statement, we adopt the following procedure.

(i) At every data point, denoted by the index $i$ and located at the momentum $r_{i}$, we consider the standard error in the lattice data for $L_{s g}$, denoted

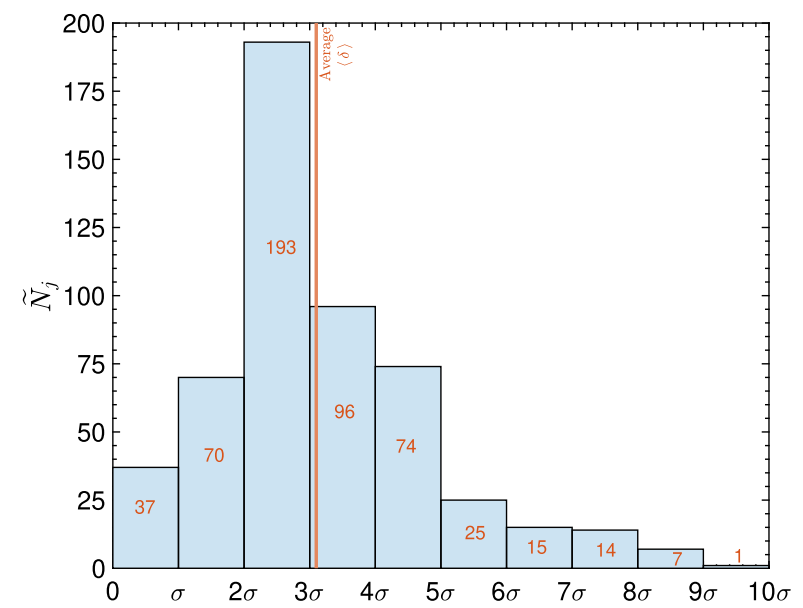

FIG. 7. Left: $\langle\delta\rangle_{k}$, the average standard deviation of all point belonging to the $k$ th bin of momentum, plotted as a function of the momentum $r$. The inset describes the definition of the local individual deviations $\rho_{i}$ and $\tau_{i}$, which are combined in a total correlated error $\sigma_{i}=\rho_{i}+\tau_{i}$, valid for any given point, as described in the text, items (i)-(iii). Right: distribution of all available points into bins of a given standard deviation. In both figures, the orange line represents the total average, $\langle\delta\rangle=3.1 \sigma$. 
by $\rho_{i}$, and the propagated error in the null hypothesis prediction, $L_{0}\left(r_{i}\right)$, denoted by $\tau_{i}$, as shown in the inset of the left panel of Fig. 7.

(ii) These errors are found to be correlated. Specifically, when using a higher $L_{s g}\left(r^{2}\right)$ as input in Eq. (6.1), we obtain a lower $L_{0}\left(r^{2}\right)$. Hence, the total error, denoted by $\sigma_{i}$, is given by $\sigma_{i}:=\rho_{i}+\tau_{i}$. Note that the $\sigma_{i}$ so defined is larger than the corresponding errors combined in quadrature, i.e., $\sqrt{\rho_{i}^{2}+\tau_{i}^{2}}$, which would be appropriate if the $\rho_{i}$ and $\tau_{i}$ were independent.

(iii) Next, we measure the distance $\Delta L_{i}:=\mid L_{0}\left(r_{i}^{2}\right)-$ $L_{s g}\left(r_{i}^{2}\right) \mid$, also shown in the inset of the left panel of Fig. 7, and then divide it by the corresponding total error, $\sigma_{i}$. The resulting ratio, $\delta_{i}:=\Delta L_{i} / \sigma_{i}$, measures the point-by-point deviation between the two curves, computed in units of the $\sigma$ (standard deviation) assigned to every given data point.

(iv) The entire momentum range considered, $[0,4.5] \mathrm{GeV}$, is divided into nine equal bins of length $0.5 \mathrm{GeV}$; thus, the $k$ th bin is defined as the interval $[0.5(k-1), 0.5 k] \mathrm{GeV}, k=1, \ldots, 9$. In addition, we denote by $N_{k}$ the total number of points in the $k$ th bin; we have $N_{k}=(52,167,157,69,40,24$, $10,7,6)$, accounting for a total of $N=532$ lattice points.

(v) Then, we compute the average value of the ratio $\delta_{i}$ within the $k$ th bin, and denote the answer by $\langle\delta\rangle_{k}$, namely

$$
\langle\delta\rangle_{k}=\frac{1}{N_{k}} \sum_{i=n_{k}+1}^{n_{k}+N_{k}} \delta_{i}, \quad \text { with } \quad n_{k}=\sum_{\ell=1}^{k-1} N_{\ell}
$$

(vi) Finally, the total average, $\langle\delta\rangle$, is defined as

$$
\langle\delta\rangle=\frac{1}{N} \sum_{i=1}^{N} \delta_{i}
$$

and furnishes a measure of the global deviation between the signal $\left[L_{s g}\left(r^{2}\right)\right]$ and the null hypothesis $\left[L_{0}\left(r^{2}\right)\right]$ curves.

The outcome of this procedure is displayed in the left panel of Fig. 7, where the quantity $\langle\delta\rangle_{k}$, obtained at step (v), is plotted for each bin. The value of $\langle\delta\rangle$, computed at step (vi), is $\langle\delta\rangle=3.1$, and is marked by the orange horizontal line.

As we can observe, the values of $\langle\delta\rangle_{k}$ for the bins with $r>1.5 \mathrm{GeV}$ are considerably higher than $\langle\delta\rangle$. In fact, for $r>2 \mathrm{GeV}$ the value of the corresponding $\langle\delta\rangle_{k}$ exceeds $5 \sigma$; however, the available points in this interval are relatively few. The sizable signal found above $2 \mathrm{GeV}$ may be understood as follows. First, near $2 \mathrm{GeV}$, the lattice curve $L_{s g}$ is the farthest away from its null hypothesis counterpart, $L_{0}$, leading to large values for the $\Delta L_{i}$ [see (iii)] in that region. Second, for $r>3 \mathrm{GeV}$, the $L_{s g}\left(r^{2}\right)$ and $L_{0}\left(r^{2}\right)$ approach each other; nevertheless, since the lattice error bars become very small in the UV, a rather strong signal emerges.

We next consider the distribution of all available points into bins of a given standard deviation, regardless of the momentum assigned to each point. The length of each bin is one $\sigma$, the $j$ th $\operatorname{bin}(j=1, \ldots 10)$ contains all points whose standard deviation lies in the interval $[j-1, j] \sigma$, and we denote the number of these points by $\tilde{N}_{j}$. The result of this grouping is shown in the right panel of Fig. 7. We observe that, the largest number of points (193) is contained in the $[2-3] \sigma$ bin, while 133 points, corresponding to $25 \%$ of the total number, are at or above the $5 \sigma$ significance level. The average of $3.1 \sigma$ is denoted by the orange vertical line.

Given that the truncation error in $\mathcal{W}\left(r^{2}\right)$ is the main uncertainty in our analysis, we end this section by considering two interesting limiting cases associated with this function.

First, given the clear proximity between $\mathbb{C}\left(r^{2}\right)$ and $\mathbb{C}_{\star}\left(r^{2}\right)$, shown in the left panel of Fig. 6 , it is tempting to ask whether a small modification in the shape of $\mathcal{W}\left(r^{2}\right)$ could make $\mathbb{C}\left(r^{2}\right)$ and $\mathbb{C}_{\star}\left(r^{2}\right)$ agree perfectly.

To that end we substitute $\mathcal{W}\left(r^{2}\right) \rightarrow \mathcal{W}_{\star}\left(r^{2}\right)$ and $\mathbb{C}\left(r^{2}\right) \rightarrow \mathbb{C}_{\star}\left(r^{2}\right)$ in Eq. (4.12) to obtain the function $\mathcal{W}_{\star}\left(r^{2}\right)$ necessary to reproduce $\mathbb{C}_{\star}\left(r^{2}\right)$. Specifically,

$\mathcal{W}_{\star}\left(r^{2}\right)=r^{2} \Delta\left(r^{2}\right)\left\{\frac{L_{s g}\left(r^{2}\right)-\mathbb{C}_{\star}\left(r^{2}\right)}{F(0)}-\tilde{Z}_{1}\left[\Delta^{-1}\left(r^{2}\right)\right]^{\prime}\right\}$.

The $\mathcal{W}_{\star}\left(r^{2}\right)$ resulting from Eq. (6.4) is shown as the purple dot-dashed curve and the associated error band in Fig. 8, where it is compared to the SDE result for $\mathcal{W}\left(r^{2}\right)$. Indeed, we observe that a minor adjustment in the shape of $\mathcal{W}\left(r^{2}\right)$ would bring $\mathbb{C}\left(r^{2}\right)$ and $\mathbb{C}_{\star}\left(r^{2}\right)$ to a perfect agreement.

Second, it is instructive to consider what would happen if the null hypothesis were valid, and all resulting mismatches were to be absorbed exclusively into a modification of $\mathcal{W}\left(r^{2}\right)$, to be denoted by $\mathcal{W}_{0}\left(r^{2}\right)$.

Setting $\mathbb{C}\left(r^{2}\right)=0$ and $\mathcal{W}\left(r^{2}\right) \rightarrow \mathcal{W}_{0}\left(r^{2}\right)$ into Eq. (4.12), we obtain

$$
\mathcal{W}_{0}\left(r^{2}\right)=r^{2} \Delta\left(r^{2}\right)\left\{\frac{L_{s g}\left(r^{2}\right)}{F(0)}-\tilde{Z}_{1}\left[\Delta^{-1}\left(r^{2}\right)\right]^{\prime}\right\} .
$$

In Fig. 8 we show $\mathcal{W}_{0}\left(r^{2}\right)$ as the green dotted curve. The band around it represents the propagated error of the lattice $L_{s g}\left(r^{2}\right)$; it is obtained by substituting $L_{s g}\left(r^{2}\right)$ in Eq. (6.5) by the $L_{s g}^{ \pm}\left(r^{2}\right)$ of Eq. (C13).

We note that the $\mathcal{W}\left(r^{2}\right)$ obtained from the SDE (blue solid curve) is comfortably separated from $\mathcal{W}_{0}\left(r^{2}\right)$. In fact, 


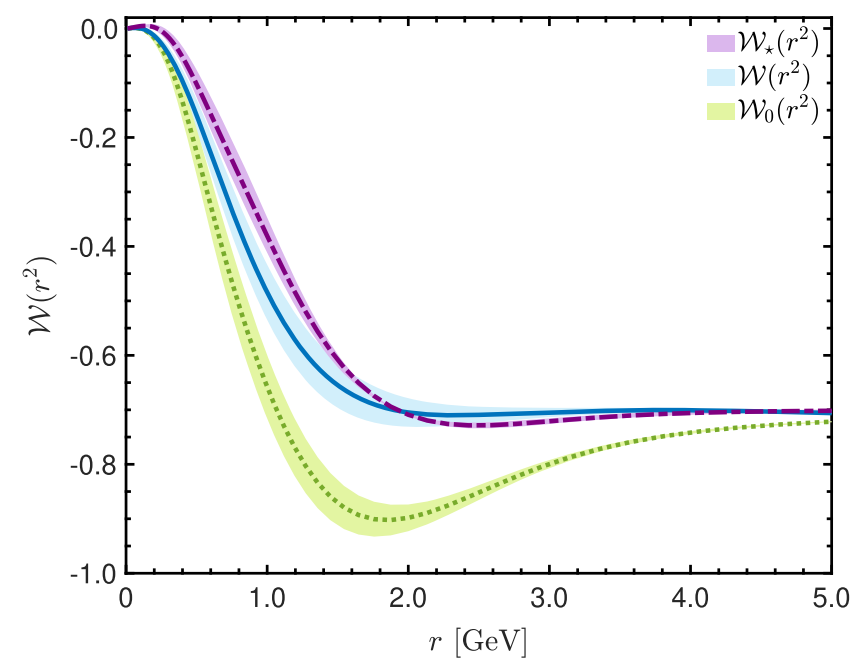

FIG. 8. Direct comparison between the SDE result for $\mathcal{W}\left(r^{2}\right)$ (blue continuous) and the $\mathcal{W}_{\star}\left(r^{2}\right)$ (purple dot-dashed) that imposes the equality between the $\mathbb{C}\left(r^{2}\right)$ obtained from the WI and the BSE. Also shown is the $\mathcal{W}_{0}\left(r^{2}\right)$ that corresponds to the null hypothesis (green dotted). The bands around each curve correspond to the propagated errors from the lattice $L_{s g}\left(r^{2}\right)$.

our attempts to obtain solutions of the SDE in the vicinity of $\mathcal{W}_{0}\left(r^{2}\right)$ have been unavailing.

\section{ADDITIONAL CONSIDERATIONS REGARDING $\mathbb{C}\left(\boldsymbol{r}^{2}\right)$ AND $\mathcal{C}\left(\boldsymbol{r}^{2}\right)$}

Given the central importance of the displacement functions $\mathbb{C}\left(r^{2}\right)$ and $\mathcal{C}\left(r^{2}\right)$ to our analysis, in this section we provide some additional information about their nature and basic properties.

(i) The numerical solution for $\mathbb{C}\left(r^{2}\right)$ obtained from the BSE, and shown in Fig. 6, saturates at a nonzero value at the origin. On the other hand, we see from

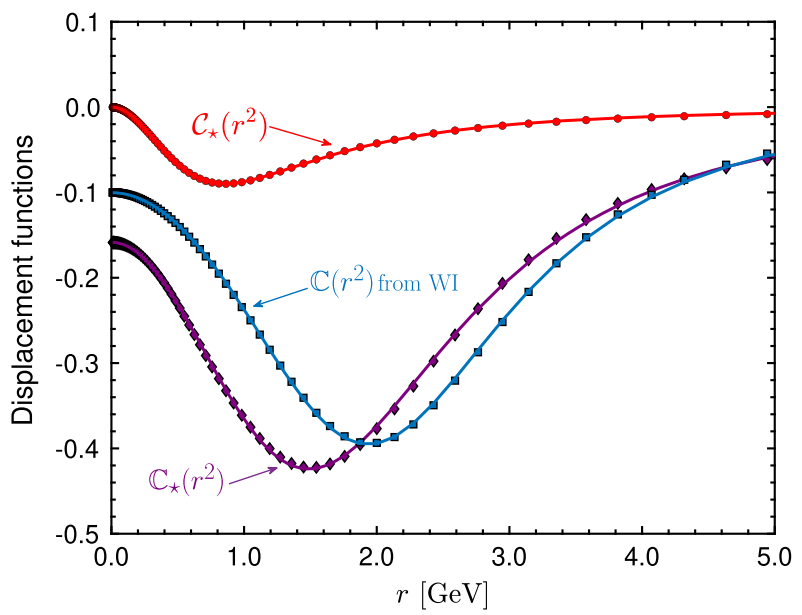

Fig. 5, as well as directly from Eq. (A4), that $\mathcal{C}(0)=0$. In fact, expanding the second line of Eq. (A4) around $r=0$, it is easily shown that

$$
\mathcal{C}\left(r^{2}\right) \sim a r^{2}
$$

for some constant $a$, while the term $\mathcal{O}(r)$ does not contribute because its angular dependence vanishes upon integration.

For asymptotically large momenta, the numerical solutions of both $\mathbb{C}\left(r^{2}\right)$ and $\mathcal{C}\left(r^{2}\right)$ tend to zero, albeit at different rates. Specifically, in both cases one finds that, as $r^{2} \rightarrow \infty$, the tails may be fitted by a common functional form $b / r^{2(1+t)}$, but with varying values for the parameters $b$ and $t$.

A functional form, that fits very accurately (see left panel of Fig. 9) the BSE results for both amplitudes and the central $\mathbb{C}\left(r^{2}\right)$ obtained from the Ward identity, is given by

$$
\begin{aligned}
& h\left(r^{2}\right)=\frac{a_{0}+a_{1}^{2} r^{2}}{1+a_{2}^{2} r^{2}+\left(a_{3}^{2} r^{2}\right)^{2+t},} \\
& \text { where } h\left(r^{2}\right) \in\left\{\mathbb{C}\left(r^{2}\right), \mathbb{C}_{\star}\left(r^{2}\right), \mathcal{C}_{\star}\left(r^{2}\right)\right\} .
\end{aligned}
$$

The parameters used for each case are given in Table I; for $\mathcal{C}_{\star}\left(r^{2}\right)$ we impose $a_{0}=0$, such that Eq. (7.1) is satisfied.

(ii) As has already been mentioned, the functions $\mathbb{C}\left(r^{2}\right)$ and $\mathcal{C}\left(r^{2}\right)$ are key ingredients in the determination of the gluon mass scale, see Eqs. (2.19) and (3.25). Due to the considerable size difference between $\mathbb{C}\left(r^{2}\right)$ and $\mathcal{C}\left(r^{2}\right)$, it is clear that the main bulk of the gluon mass scale will be obtained from Eq. (3.25), where $\mathbb{C}\left(r^{2}\right)$ will enter directly after use of Eq. (3.26). Even though a full analysis requires knowledge of the

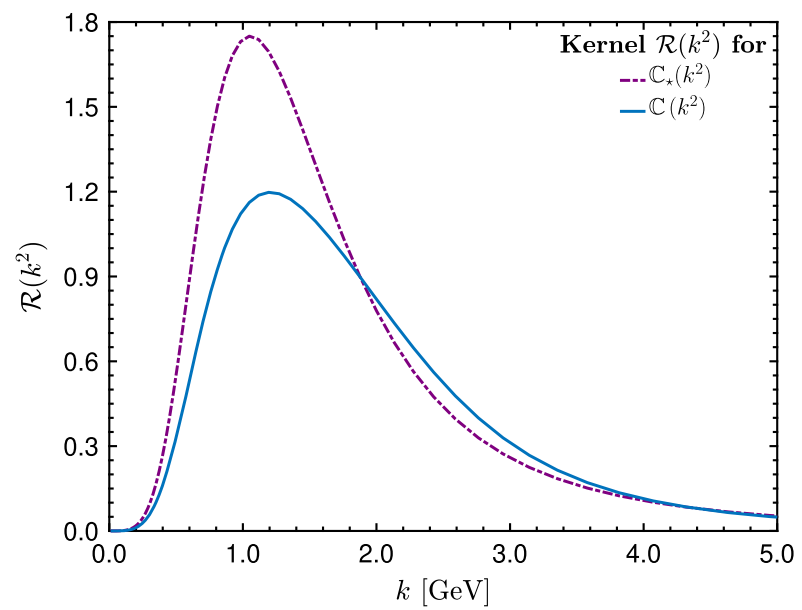

FIG. 9. Left: fits for the BSE amplitudes $\mathcal{C}_{\star}\left(r^{2}\right)$ (red continuous curve) and $\mathbb{C}_{\star}\left(r^{2}\right)$ (purple continuous) and for the central curve of $\mathbb{C}\left(r^{2}\right)$ (blue continuous line) obtained from the WI shown in the left panel of Fig. 6. The functional form is given by Eq. (7.2). Right: strength of the kernel $\mathcal{R}\left(k^{2}\right)$ of Eq. (7.3) computed using $\mathbb{C}_{\star}\left(k^{2}\right)$, (purple line) and $\mathbb{C}\left(r^{2}\right)$ (blue curve) shown in the left panel. 
TABLE I. The values of the fitting parameters appearing in Eq. (7.2), for each of the functions $\mathbb{C}\left(r^{2}\right), \mathbb{C}_{\star}\left(r^{2}\right)$, and $\mathcal{C}_{\star}\left(r^{2}\right)$.

\begin{tabular}{lccccc}
\hline \hline & $a_{0}$ & $a_{1}^{2}\left[\mathrm{GeV}^{-2}\right]$ & $a_{2}^{2}\left[\mathrm{GeV}^{-2}\right]$ & $a_{3}^{2}\left[\mathrm{GeV}^{-2}\right]$ & $t$ \\
\hline $\mathbb{C}\left(r^{2}\right)$ & -0.100 & -0.162 & 0.092 & 0.197 & 0.68 \\
$\mathbb{C}_{\star}\left(r^{2}\right)$ & -0.158 & -0.397 & 0.456 & 0.321 & 0.45 \\
$\mathcal{C}_{\star}\left(r^{2}\right)$ & 0 & -0.473 & 2.71 & 1.29 & 0.12 \\
\hline \hline
\end{tabular}

renormalized quantity $Y\left(k^{2}\right)$ (or an equivalent diagrammatic expansion, see [138]), one may get a general idea of how the support of $\mathbb{C}\left(r^{2}\right)$ is distributed in Eq. (3.25) by considering the natural combination that appears therein, namely

$$
\mathcal{R}\left(k^{2}\right):=\mathcal{Z}^{2}\left(k^{2}\right)\left|\mathbb{C}\left(k^{2}\right)\right|,
$$

where $\mathcal{Z}\left(k^{2}\right):=k^{2} \Delta\left(k^{2}\right)$ is the dressing function of the gluon.

For the numerical evaluation of $\mathcal{R}\left(k^{2}\right)$ we use the purple and blue curves shown in the left panel of Fig. 9. The results obtained are shown in the right panel of Fig. 9; it is clear that in both cases the support of $\mathcal{R}\left(k^{2}\right)$ peaks at around $1.2 \mathrm{GeV}$, which is the typical scale for the onset of nonperturbative effects.

(iii) It is clear from the left panel of Fig. 9 that, for large momenta, $\mathbb{C}\left(r^{2}\right)$ approaches zero rather slowly, maintaining a considerable size within the region of 2-3 GeV. In general terms, this persistence of the signal suggests that the underlying mass generating mechanism furnishes more support in the region of intermediate momenta rather than in the deep infrared, a pattern already familiar from the quark sector of QCD [139,140]. A possible explanation for this particular feature may be obtained within the context of the BSE that determines $\mathbb{C}\left(r^{2}\right)$ [see Sec. V and Appendix A].

Specifically, in the BSE of (A4) we turn off the subleading term $\mathcal{C}\left(r^{2}\right)$, converting the system into a single integral equation for $\mathbb{C}\left(r^{2}\right)$, whose kernel is give by

$$
\begin{aligned}
\mathcal{K}_{\mathbb{C}}\left(r^{2}, k^{2}\right) & =r k^{3} \Delta^{2}\left(k^{2}\right) \int_{0}^{\pi} d \phi s_{\phi}^{2} c_{\phi} \Delta(u) \mathcal{N}\left(r^{2}, k^{2}, u\right), \\
u & =(r-k)^{2},
\end{aligned}
$$

where the constant $\lambda$ and the integration over $k^{2}$ have been omitted.

The kernel $\mathcal{K}_{\mathbb{C}}\left(r^{2}, k^{2}\right)$ is a function of two variables, and may be represented by a 3D surface. However, we find it more instructive to plot "slices" of it, fixing five values of the momentum $r$, as can be

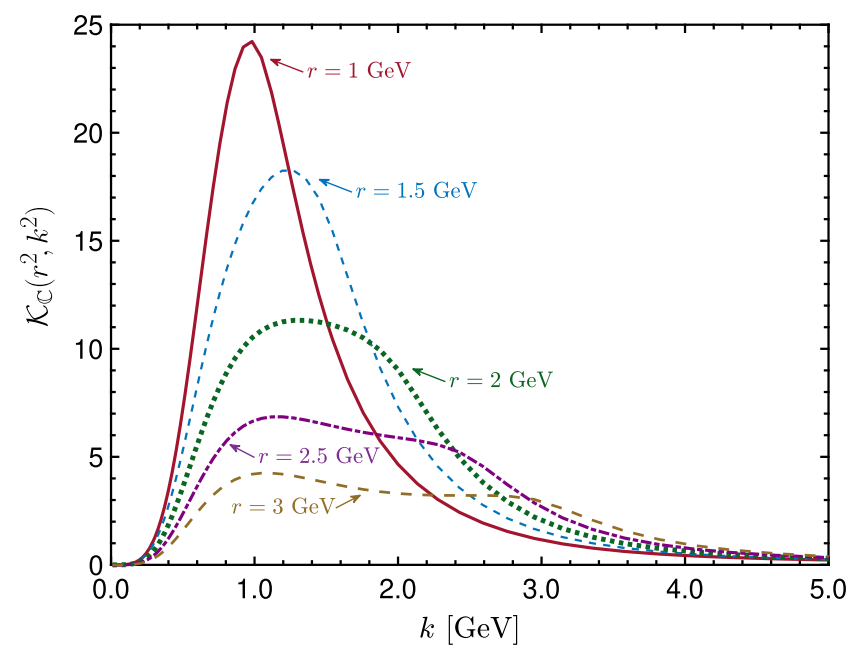

FIG. 10. The kernel $\mathcal{K}_{\mathbb{C}}\left(r^{2}, k^{2}\right)$ defined in Eq. (7.4) for five values of $r$. The increase in the value of $r$ produces a flattening in the kernel.

seen in Fig. 10. The succession of the curves indicates that, while the corresponding peaks decrease as $r$ increases, the curves become wider and their support gets shifted towards higher momenta. Thus, the strength of the kernel peters out rather slowly, causing the solution for $\mathbb{C}\left(r^{2}\right)$ to retain a considerable size past $2 \mathrm{GeV}$.

(iv) In the present work, all procedures furnishing $\mathbb{C}\left(r^{2}\right)$ have been implemented using as inputs the so-called "decoupling" SDE solutions, found in the lattice simulations cited throughout the text. The resulting $\mathbb{C}\left(r^{2}\right)$ shown in Fig. 6 acquires its highest (absolute) value around $1.5-2 \mathrm{GeV}$, being considerably suppressed at the origin. In fact, the results from the WI determination (blue band in Fig. 6) are marginally compatible with $\mathbb{C}(0)=0$, a behavior that is reminiscent of the so-called "scaling" solutions (see, e.g., [141]), specific to the "infrared ghost dominance" scenario [142].

In the simplified case of a single momentum scale, $p^{2}$, this later type of solution is of the general form (as $p^{2} \rightarrow 0$ )

$$
\Gamma^{n, m}\left(p^{2}\right) \sim\left(p^{2}\right)^{(n-m) \kappa},
$$

where the $\Gamma^{n, m}\left(p^{2}\right)$ denote all one-particle irreducible Greens functions with $n$ external ghost and antighost legs, and $m$ gluon legs. The exact value of the parameter $\kappa$ depends on the details of the SDE truncation, but it is rather close to $\kappa=0.6$. Note that, in the notation of the present paper, $\Gamma^{0,2}\left(p^{2}\right)=$ $\Delta^{-1}\left(p^{2}\right)$; so, according to Eq. (7.5), the scaling gluon propagator vanishes at the origin. Similarly, the 
scaling ghost dressing function diverges at the origin, and so do the three- and four-gluon vertex functions. It is important to emphasize that, despite their clear discrepancies in the deep infrared, the scaling and decoupling solutions practically coincide for momenta higher than a few hundred $\mathrm{MeV}$ (see, e.g., [143]). Let us also point out that the Schwinger mechanism has been recently implemented in the context of the scaling solutions [94]. In this case, the generation of the mass scale does not affect the deep infrared regime of the corresponding Green's functions, but accounts for their considerable support in the intermediate range of momenta.

Finally, one may hypothesize on the properties of $\mathbb{C}\left(r^{2}\right)$ if scaling solutions were used for its determination. Given the observations made above, it is reasonable to expect that its qualitative behavior would remain unaffected for momenta larger than a few hundred $\mathrm{MeV}$, while differences would manifest themselves in the deep infrared. In fact, it is perfectly plausible that $\mathbb{C}\left(r^{2}\right)$ might vanish at the origin, exactly as $\mathcal{C}\left(r^{2}\right)$ does.

\section{DISCUSSION AND CONCLUSIONS}

In the present work we have investigated in detail a characteristic feature that is intimately linked with the onset of the Schwinger mechanism in QCD, and the ensuing emergence of an effective gluon mass scale. The action of this mechanism relies on the inclusion of massless longitudinal poles in the fundamental vertices of the theory, which participate nontrivially in the realization of the corresponding STIs. This, in turn, causes a distinct displacement to the WI satisfied by the pole-free part of the vertices involved, quantified by the function $\mathbb{C}\left(r^{2}\right)$, which is formally identical to the bound-state wave function that governs the dynamical formation of the massless poles. We have computed $\mathbb{C}\left(r^{2}\right)$ for the case of the three-gluon vertex in two distinct ways: by solving the corresponding BSE and by appropriately combining the ingredients appearing in the non-Abelian WI. In both cases we have relied predominantly on results obtained from lattice simulations, with the exception of the special function $\mathcal{W}\left(r^{2}\right)$, which was determined from an SDE. The results found for $\mathbb{C}\left(r^{2}\right)$ are clearly nonvanishing and in excellent mutual agreement, providing additional support to the details of the general dynamical picture put forth in a series of articles. In particular, the dual role played by $\mathbb{C}\left(r^{2}\right)$ is especially noteworthy, hinting towards deeper connections that have yet to be unraveled.

It is important to stress that the computation of the null hypothesis, presented in Sec. VI, proceeds by assuming that all inputs of Eq. (6.1) retain their known form; in particular, salient features, such as the saturation of the gluon propagator and the ghost dressing function, persist unaltered. In that sense, this specific implementation probes the compatibility between the lattice results and the absence of a displacement in the non-Abelian WI of the three-gluon vertex. Seen from this point of view, one might state that this particular possibility is excluded at the level of $3.1 \sigma$.

As mentioned in Appendix A, the value of $\alpha_{s}$ that corresponds to the eigenvalue of the system is $\alpha_{s}=0.63$, which is considerably different from the value $\alpha_{s}=0.27$ found within the "asymmetric" MOM scheme that we employ. The discrepancy may be interpreted as a truncation artifact, given that the corresponding BSE kernels has been approximated by their one-particle exchange diagrams, as depicted in Fig. 4. In addition, the expressions employed for the fully dressed vertices comprising these kernels contain a certain amount of uncertainty. Quite interestingly, a preliminary numerical exploration indicates that minor modifications of the kernel affect the value of $\alpha_{s}$ considerably, without practically modifying the form of the solution found for $\mathbb{C}_{\star}\left(r^{2}\right)$ and $\mathcal{C}_{\star}\left(r^{2}\right)$. This observation suggests that, while the decrease of $\alpha_{s}$ towards its MOM value may require a more refined knowledge of the corresponding BSE kernels, the obtained solutions should be considered as fairly reliable.

The proximity between the $\mathcal{W}\left(r^{2}\right)$, computed in Appendix B, and the $\mathcal{W}_{\star}\left(r^{2}\right)$ obtained from Eq. (6.4), suggest that minor modifications of the inputs used for the SDE of Eq. (B5) might lead to an even better coincidence. In this context, it is interesting to point out that the determination of the transverse form factors $Y_{i}$ [see item (iii) in Appendix B] is subject to a considerable uncertainty, originating from the approximations implemented to the complicated SDE satisfied by the three-gluon vertex (Fig. 6 in [106]). Given the relevance of $\mathcal{W}\left(r^{2}\right)$ for the systematic scrutiny of the Schwinger mechanism, as exposed in the present work, it may be worthwhile revisiting this particular computation.

As mentioned below Eq. (3.8), the Schwinger mechanism induces poles also in the ghost-gluon kernel, $H_{\mu \nu}(r, q, p)$. This may be understood qualitatively by considering the diagrams $\left(d_{1}\right)$ and $\left(d_{2}\right)$ in Fig. 12: the fully dressed ghost-gluon and three-gluon vertices (with Lorentz index $\nu$ and incoming momentum $p$ ) contain poles, which are transmitted to the form factors of $H_{\mu \nu}(r, q, p)$ associated with the tensorial structures $p_{\nu} p_{\mu}$ and $p_{\nu} r_{\mu}$. It would be important to compute in detail the pole structure of $H_{\mu \nu}(r, q, p)$, especially in view of the STI $r^{\mu} H_{\mu \nu}(r, q, p)=\Gamma_{\nu}(p, r, q)$, which links nontrivially the form factors of $H_{\mu \nu}(p, r, q)$ and $\Gamma_{\mu}(p, r, q)$, in general, and the corresponding pole terms, in particular. Specifically, one may explore how accurately the appropriate combination of pole terms coming from $H_{\mu \nu}(p, r, q)$ will reproduce 
the corresponding term contained in $\Gamma_{\mu}(p, r, q)$. We hope to undertake such a study in the near future.

The generation of massless poles in the ghost correlation functions and kernels, such as $\Gamma_{\nu}(p, r, q)$ and $H_{\mu \nu}(r, q, p)$, may herald a deeper connection with the nonperturbative BRST quartet mechanism mentioned earlier. In particular, the dynamical realization of the quartet mechanism put forth in [121] relies on the formation of massless ghostgluon bound states, which would manifest themselves in the ghost-gluon four-point kernel that enters in the corresponding BSE (see Fig. 1 in [121]). Evidently this issue deserves detailed scrutiny, both from the point of view of the decoupling as well as the scaling solutions. Such a study could be particularly revealing, establishing the compatibility and harmonious interplay between two key nonperturbative mechanism operating in the gauge sector of QCD.

The scale ambiguity associated with the BSE amplitudes $\mathbb{C}\left(r^{2}\right)$ and $\mathcal{C}\left(r^{2}\right)$ results from considering only the leading order terms of the BSEs in an expansion around $q=0$, which furnishes homogeneous linear equations. In general kinematics, however, the presence of inhomogeneous terms in the BSEs resolves this ambiguity. As such, the scales of $\mathbb{C}\left(r^{2}\right)$ and $\mathcal{C}\left(r^{2}\right)$ can be fixed by taking the $q=0$ limit of the solution of the corresponding inhomogeneous BSEs, treated beyond leading order in $q$. In the context of conventional bound states, this procedure is well understood [144] and explicit scale-setting equations have been derived, which are sometimes referred to as "canonical normalization condition" $[28,145]$. It is our intention to pursue this point in an upcoming study, and settle dynamically the scale of the corresponding solutions.

\section{ACKNOWLEDGMENTS}

The work of A. C. A. is supported by the Brazilian CNPq Grants No. 307854/2019-1 and No. 464898/2014-5 (INCTFNA). A.C. A. and M. N.F. also acknowledge financial support from the FAPESP Projects No. 2017/05685-2 and No. 2020/12795-1, respectively. J. P. is supported by the Spanish AEI-MICINN Grant No. PID2020-113334GBI00/AEI/10.13039/501100011033, and the grant Prometeo/ 2019/087 of the Generalitat Valenciana.

\section{APPENDIX A: TECHNICAL DETAILS ON THE BSE SYSTEM}

In this appendix we present details related to the numerical treatment of the BSE system formed by $\mathbb{C}\left(r^{2}\right)$ and $\mathcal{C}\left(r^{2}\right)$, shown in Fig. 4.

For the Bose symmetric three-gluon vertex appearing in the diagrams of Fig. 4 we employ the tensor basis of BallChiu [146,147],

$\Gamma^{\alpha \mu \nu}(q, r, p)=\sum_{i=1}^{10} X_{i}(q, r, p) \ell_{i}^{\alpha \mu \nu}+\sum_{i=1}^{4} Y_{i}(q, r, p) t_{i}^{\alpha \mu \nu}$

where the explicit form of the basis tensors $\ell_{i}^{\alpha \mu \nu}$ and $t_{i}^{\alpha \mu \nu}$ is given in Eqs. (3.4) and (3.6) of [147]. At tree level, $X_{1}^{(0)}=X_{4}^{(0)}=X_{7}^{(0)}=1$, while all other $X_{i}^{(0)}$ and $Y_{i}^{(0)}$ vanish.

It is convenient to introduce the transversely projected vertex, $\bar{\Gamma}_{\alpha \mu \nu}(q, r, p)$, defined as

$\bar{\Gamma}_{\alpha \mu \nu}(q, r, p):=P_{\alpha}^{\alpha^{\prime}}(q) P_{\mu}^{\mu^{\prime}}(r) P_{\nu}^{\nu^{\prime}}(p) \Gamma_{\alpha^{\prime} \mu^{\prime} \nu^{\prime}}(q, r, p)$.

Similarly, the tensorial decomposition of the vertex $\Gamma_{\mu}(q, r, p)$ is given by

$$
\Gamma_{\mu}(q, r, p)=r_{\mu} B_{1}(r, p, q)+q_{\mu} B_{2}(r, p, q)
$$

at tree level, $B_{1}^{(0)}=1$ and $B_{2}^{(0)}=0$. The ghost-antighost symmetry of $\Gamma_{\mu}(q, r, p)$ in the Landau gauge implies that $B_{1}(r, p, q)=B_{1}(p, r, q)$.

Next, we pass to Euclidean space and employ spherical coordinates. For convenience, we define the variables $x:=r^{2}, y:=k^{2}$, and $u:=(r-k)^{2}=x+y-2 \sqrt{x y} c_{\phi}$, with $\phi$ denoting the angle between the momenta $k$ and $r$, while $s_{\phi}:=\sin \phi, c_{\phi}:=\cos \phi$. Furthermore, we parametrize scalar form factors, such as $B_{1}(q, r, p)$, in terms of the squares of their first two arguments and the angle between them, e.g., $B_{1}(-r, k, r-k) \rightarrow B_{1}(x, y, \pi-\phi)$. Then, the final set of BSEs reads

$$
\begin{aligned}
& \mathbb{C}(x)=\lambda\left[\int_{0}^{\infty} d y y \sqrt{x y} \Delta^{2}(y) \mathbb{C}(y) \int_{0}^{\pi} d \phi s_{\phi}^{2} c_{\phi} \Delta(u) \mathcal{N}(x, y, u)-2 \int_{0}^{\infty} d y \sqrt{\frac{y}{x}} F^{2}(y) \mathcal{C}(y) \int_{0}^{\pi} d \phi s_{\phi}^{4} c_{\phi} \frac{F(u)}{u} B_{1}^{2}(u, y, \chi)\right], \\
& \mathcal{C}(x)=3 \lambda\left[\int_{0}^{\infty} d y y \sqrt{x y} \Delta^{2}(y) \mathbb{C}(y) \int_{0}^{\pi} d \phi s_{\phi}^{4} c_{\phi} \frac{F(u)}{u} B_{1}^{2}(x, u, \theta)+\int_{0}^{\infty} d y \sqrt{x y} F^{2}(y) \mathcal{C}(y) \int_{0}^{\pi} d \phi s_{\phi}^{4} c_{\phi} \frac{\Delta(u)}{u} B_{1}^{2}(x, y, \pi-\phi)\right] .
\end{aligned}
$$

In the above equation, 


$$
\lambda:=\frac{\alpha_{s} C_{\mathrm{A}}}{12 \pi^{2}}
$$

$\mathcal{N}(x, y, u):=-\frac{1}{x}\left[\bar{\Gamma}_{\alpha \beta \gamma}(-r, k, r-k) \bar{\Gamma}^{\alpha \beta \gamma}(-r, k, r-k)\right]_{\mathrm{E}}$,

where $\alpha_{s}:=g^{2} / 4 \pi$. The angles $\chi$ and $\theta$ are given by

$$
\begin{aligned}
& \chi=\cos ^{-1}\left(\frac{\sqrt{x} \cos \phi-\sqrt{y}}{\sqrt{u}}\right), \\
& \theta=\cos ^{-1}\left(\frac{\sqrt{y} \cos \phi-\sqrt{x}}{\sqrt{u}}\right) .
\end{aligned}
$$

Finally,

Then, we obtain

$$
\begin{aligned}
\mathcal{N}(x, y, u)= & \frac{s_{\phi}^{2}}{2 u^{2} x^{2} y}\left\{u T_{1}\left[T_{1}\left((u-x-y)^{2}+2 x y\right)+4 x y\left(T_{2}+T_{3}\right)+4 T_{4}(u-x-y)\right]\right. \\
& \left.+x T_{2}\left[T_{2}\left((u-x+y)^{2}+2 u y\right)\right)+4 u y T_{3}-4 T_{4}(u-x+y)\right] \\
& \left.+y T_{3}\left[T_{3}\left((u+x-y)^{2}+2 u x\right)-4 T_{4}(u+x-y)\right]+4 T_{4}^{2}(u+x+y)\right\},
\end{aligned}
$$

where

$$
\begin{aligned}
& T_{1}=-u\left(X_{1}-X_{4}-X_{7}+x y Y_{1}\right)-x\left(X_{1}-X_{4}+X_{7}-2 y X_{3}\right)-y\left(X_{1}+X_{4}-X_{7}\right), \\
& T_{2}=u\left(X_{1}-X_{4}-X_{7}+2 y X_{6}-x y Y_{2}\right)+x\left(X_{1}-X_{4}+X_{7}\right)-y\left(X_{1}+X_{4}-X_{7}\right), \\
& T_{3}=u\left(X_{1}-X_{4}-X_{7}+2 x X_{9}-x y Y_{3}\right)-x\left(X_{1}-X_{4}+X_{7}\right)+y\left(X_{1}+X_{4}-X_{7}\right), \\
& T_{4}=\frac{1}{2}\left\{u^{2}\left(X_{1}-X_{4}-X_{7}\right)-(x-y)\left[x\left(X_{1}-X_{4}+X_{7}\right)-y\left(X_{1}+X_{4}-X_{7}\right)\right]+2 u\left[x\left(X_{7}-y Y_{4}\right)+y X_{4}\right]\right\},
\end{aligned}
$$

and we suppress the functional dependence $X_{i} \equiv X_{i}(x, y, \pi-\phi)$ and $Y_{j} \equiv Y_{j}(x, y, \pi-\phi)$.

For the numerical evaluation of Eq. (A4), in addition to the lattice propagators given in Appendix $\mathrm{C}$, we need $B_{1}$, together with all $X_{i}$ and $Y_{i}$ that comprise $\mathcal{N}(x, y, u)$, in general kinematics. These form factors are obtained as follows:

(i) For the $B_{1}$ we employ recent results (see Fig. 6 of [130]), obtained from an SDE analysis that uses as inputs lattice data that have been cured from volume and discretization artifacts.

(ii) The $X_{i}$ are obtained from the nonperturbative generalization of the Ball-Chiu solution; the relevant formulas are given in Eq. (3.11) of [147], and involve the ghost dressing function, the kinetic term of the gluon propagator, and certain components of the ghost-gluon kernel. Note that the inputs have been calibrated to exactly reproduce the lattice projection $L_{s g}\left(r^{2}\right)$, through the relation

$$
L_{s g}\left(r^{2}\right)=X_{1}\left(r^{2}, r^{2}, \pi\right)-r^{2} X_{3}\left(r^{2}, r^{2}, \pi\right) .
$$

In this indirect way, the error bars assigned to the lattice calculation of $L_{s g}\left(r^{2}\right)$, encompassed by the functions $L_{s g}^{ \pm}\left(r^{2}\right)$ of Eq. (C13), find their way into our BSE determination of $\mathbb{C}\left(r^{2}\right)$ and $\mathcal{C}\left(r^{2}\right)$, giving rise to the errors band indicated in Fig. 6 .

(iii) For the transverse components $Y_{i}$, which cannot be deduced from the fundamental STIs, we resort to a SDE determination, along the lines of the analysis presented in [106]; see, in particular, Fig. 6 therein.

(iv) The results for $X_{1}\left(q^{2}, r^{2}, \theta\right), q r X_{3}\left(q^{2}, r^{2}, \theta\right)$, $q^{2} r^{2} Y_{1}\left(q^{2}, r^{2}, \theta\right)$, and $q r Y_{4}\left(q^{2}, r^{2}, \theta\right)$ are shown in Fig. 11, for the special case $\theta=2 \pi / 3 ; q$ and $r$ denote now the magnitudes of the corresponding Euclidean momenta.

(v) By virtue of the Bose symmetry of the three-gluon vertex, the remaining form factors of the three-gluon vertex entering in Eq. (A7) can be obtained from those shown in Fig. 11 by appropriate permutations of their arguments, as explained in [147]. 

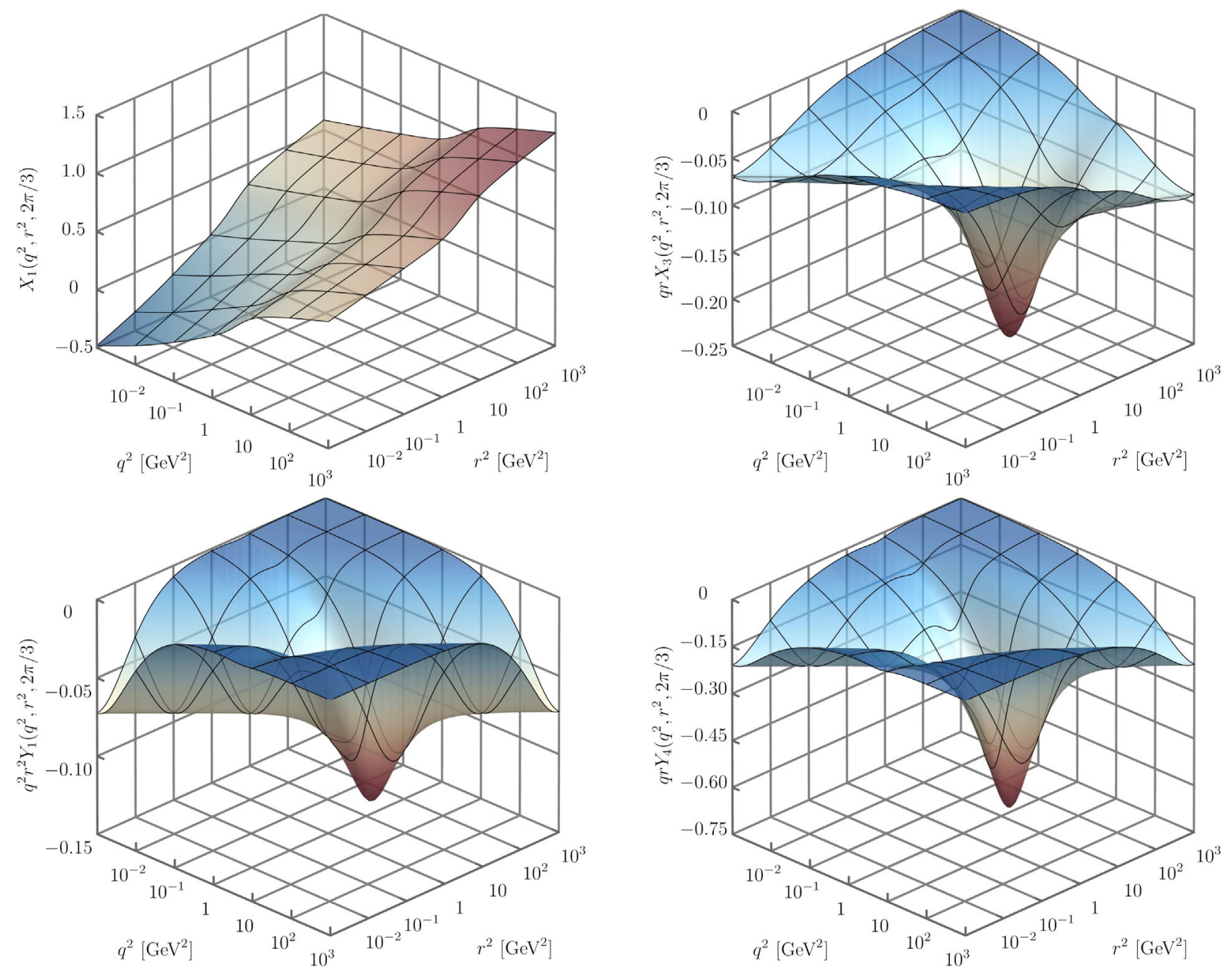

FIG. 11. The three-gluon vertex form factors used in the BSE system: $X_{1}\left(q^{2}, r^{2}, 2 \pi / 3\right)$ (top left), $q r X_{3}\left(q^{2}, r^{2}, 2 \pi / 3\right)$ (top right), $q^{2} r^{2} Y_{1}\left(q^{2}, r^{2}, 2 \pi / 3\right)$ (bottom left), and $q r Y_{4}\left(q^{2}, r^{2}, 2 \pi / 3\right)$ (bottom right).

Employing the ingredients described above, we solve the coupled system of BSEs of Eq. (A4) numerically, obtaining the $\mathbb{C}\left(r^{2}\right)$ and $\mathcal{C}\left(r^{2}\right)$ shown in Fig. 5, together with the corresponding error estimates.

Since Eq. (A4) does not have an inhomogeneous term and is linear in $\mathbb{C}\left(r^{2}\right)$ and $\mathcal{C}\left(r^{2}\right)$, it corresponds to an eigenvalue problem. The resulting eigenvalues correspond to $\alpha_{s}=0.63 \mp 0.05$, with signs opposite to those of the $L_{s g}^{ \pm}\left(r^{2}\right)$ error bands, i.e., using $X_{i}$ corresponding to a higher $L_{s g}\left(r^{2}\right)$ leads to a smaller $\alpha_{s}$. In these results, the overall constant was determined by matching the BSE prediction for $\mathbb{C}\left(r^{2}\right)$ to the result obtained from the WI, as explained in Sec. VI.

\section{APPENDIX B: COMPUTATION OF $\mathcal{W}\left(r^{2}\right)$}

The function $\mathcal{W}\left(r^{2}\right)$ is a central ingredient for our analysis, whose determination proceeds through the study of the corresponding SDE. In this appendix we present the technical details related to this calculation, and discuss the validity of our approximations.

\section{SDE, inputs, and solution}

The starting point of our determination of $\mathcal{W}\left(r^{2}\right)$ is the $\mathrm{SDE}$ for the ghost-gluon scattering kernel, $H_{\mu \nu}(r, q, p)$, shown in Fig. 12, which is truncated at the one-loop dressed level, retaining only diagrams $\left(d_{1}\right)$ and $\left(d_{2}\right)$. In the Landau gauge it is immediate to factor out of these two diagrams the ghost momentum $q$, in order to obtain $K_{\mu \nu \alpha}(r, q, p)$, in accordance with Eq. (3.16). Finally, recalling Eq. (3.18), $\mathcal{W}\left(r^{2}\right)$ is obtained by isolating the $g_{\mu \nu} r_{\alpha}$ form factor of $K_{\mu \nu \alpha}(r, 0,-r)$ and using Eq. (4.11).

Since a detailed derivation and renormalization of the $\mathcal{W}\left(r^{2}\right)$ equation has been carried out in Sec. 6 of Ref. [108], here we only collect the main results. Specifically, we obtain the general expression

$$
\mathcal{W}\left(r^{2}\right)=\mathcal{W}_{1}\left(r^{2}\right)+\mathcal{W}_{2}\left(r^{2}\right)
$$




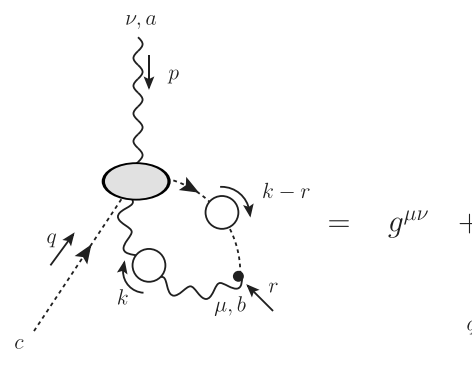

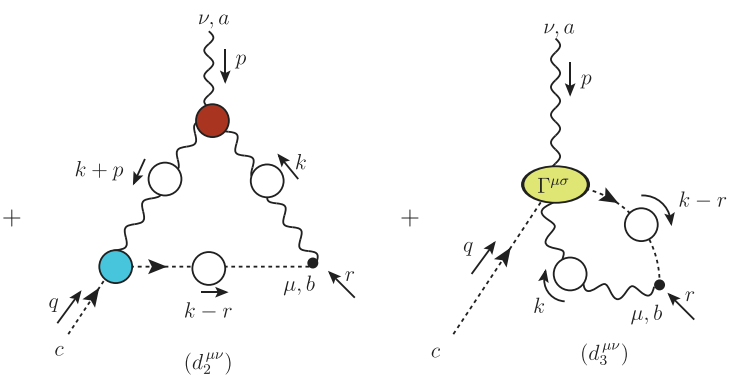

FIG. 12. The SDE satisfied by the ghost-gluon scattering kernel, $H_{\mu \nu}(r, q, p)$.

where $\mathcal{W}_{1}$ and $\mathcal{W}_{2}$ denote the contributions from diagrams $\left(d_{1}\right)$ and $\left(d_{2}\right)$ of Fig. 12, respectively, given by

$$
\begin{aligned}
& \mathcal{W}_{1}\left(r^{2}\right)=-\frac{i g^{2} C_{\mathrm{A}} \tilde{Z}_{1}}{6} \int_{k} \Delta\left(k^{2}\right) D\left(k^{2}\right) D\left(t^{2}\right) B_{1}(t, k,-r) B_{1}(k, 0,-k)(r \cdot k) f(r, k), \\
& \mathcal{W}_{2}\left(r^{2}\right)=-\frac{i g^{2} C_{\mathrm{A}} \tilde{Z}_{1}}{6} \int_{k} \Delta\left(k^{2}\right) \Delta\left(t^{2}\right) D\left(t^{2}\right) B_{1}(t, 0,-t) r^{\alpha} \bar{\Gamma}_{\mu \alpha}^{\mu}(-r, k, t),
\end{aligned}
$$

where

$$
f(r, k):=1-\frac{(k \cdot r)^{2}}{k^{2} r^{2}}
$$

above Eq. (A4). Then, using the Ball-Chiu tensor basis of Eq. (A1) for the three-gluon vertex, we obtain

$$
\mathcal{W}(x)=\mathcal{W}_{1}(x)+\mathcal{W}_{2}(x)
$$

with

$$
\begin{aligned}
& \mathcal{W}_{1}(x)=\lambda \tilde{Z}_{1} \int_{0}^{\infty} d y \sqrt{x y} \Delta(y) F(y) B_{1}(y, 0,0) \int_{0}^{\pi} d \phi c_{\phi} s_{\phi}^{4} \frac{F(u)}{u} B_{1}(u, y, \chi), \\
& \mathcal{W}_{2}(x)=-2 \lambda \tilde{Z}_{1} \int_{0}^{\infty} d y y \sqrt{x y} \Delta(y) \int_{0}^{\pi} d \phi s_{\phi}^{4} \Delta(u) B_{1}(u, 0,0) \frac{F(u)}{u^{2}} \mathcal{K}(x, y, u),
\end{aligned}
$$

where $\lambda$ is defined in Eq. (A5), $\chi$ in Eq. (A6), and we define the kernel $\mathcal{K}(x, y, u)$ as

$$
\begin{aligned}
\mathcal{K}(x, y, u):= & \sqrt{x y}\left(c_{\phi}^{2}+2\right) X_{1}+\left(y-c_{\phi} \sqrt{x y}\right) c_{\phi} X_{4}+\left(x-c_{\phi} \sqrt{x y}\right) c_{\phi} X_{7}-3 c_{\phi} x y X_{3} \\
& -c_{\phi} y u X_{6}-c_{\phi} x u X_{9}+\frac{1}{2} c_{\phi} x y u\left(3 Y_{1}+Y_{2}+Y_{3}\right)-u \sqrt{x y} Y_{4},
\end{aligned}
$$

where, again, $X_{i} \equiv X_{i}(x, y, \pi-\phi)$ and $Y_{j} \equiv Y_{j}(x, y, \pi-\phi)$.

Using the Bose symmetry relations involving permutations of arguments of the $X_{i}$ and $Y_{i}$, given by Eqs. (3.7) to (3.10) of [147], it is possible to show that $\mathcal{K}(x, y, u)$ is symmetric under the exchange of $x \leftrightarrow y$.

Then, for the gluon propagator $\Delta\left(r^{2}\right)$ and the ghost dressing function $F\left(r^{2}\right)$ we use the fits presented in Appendix $\mathrm{C}$, while for the vertex form factors, $B_{1}, X_{i}$, and $Y_{i}$ we use the same inputs employed for the solution of the BSE of Eq. (A4). Note that, as mentioned in Appendix $\mathrm{C}$, all inputs are renormalized within the "asymmetric" MOM scheme, at the renormalization point $\mu=4.3 \mathrm{GeV}$, for which $\alpha_{s}=0.27$.

With these ingredients, we obtain for $\mathcal{W}\left(r^{2}\right)$ the blue continuous line shown in the right panel of Fig. 13. The blue 

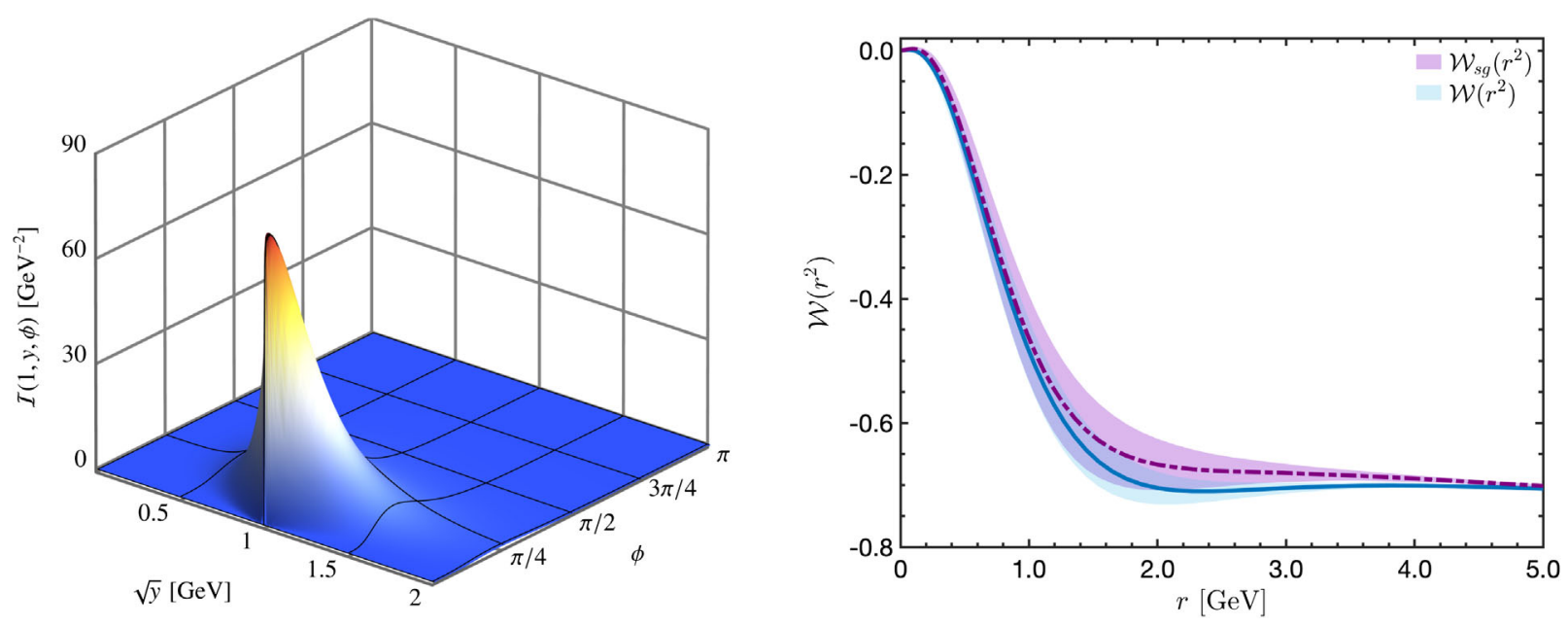

FIG. 13. Left: integrand of $\mathcal{W}_{2}(x), \mathcal{I}(x, y, \phi)$, given by Eq. (B7), for $x=1 \mathrm{GeV}^{2}$. Note that its maximum occurs when $y$ is first set to $y=x=1 \mathrm{GeV}$ and then $\phi=0$, corresponding to $u=0 . \mathcal{I}(x, y, \phi)$ is intensely peaked around the maximum, dropping rapidly to zero away from it. Right: comparison of $\mathcal{W}\left(r^{2}\right)$, obtained with Eq. (B4) (blue solid line), to $\mathcal{W}_{s g}\left(r^{2}\right)$, obtained with the replacement (B12) into Eq. (B4) (purple dot-dashed line). The bands correspond to propagated error from the lattice $L_{s g}\left(r^{2}\right)$ of [106].

band around it corresponds to an error propagated from the uncertainty in the lattice $L_{s g}\left(r^{2}\right)$, through the same procedure explained in item (ii) of Sec. A.

Given that $\mathcal{W}\left(r^{2}\right)$ is one of the main ingredients in the analysis of Eq. (4.12), it is important to consider in more detail the uncertainty in our SDE determination of this function. It turns out that the contribution $\mathcal{W}_{1}\left(r^{2}\right)$ of Eq. (B5) is negligible in comparison to $\mathcal{W}_{2}\left(r^{2}\right)$ (see Fig. 7 of [108]), except for $r<0.5 \mathrm{GeV}$, where $\mathcal{W}_{2}\left(r^{2}\right)$ decreases significantly. Furthermore, diagram $\left(d_{3}\right)$ of Fig. 12, with the four-particle correlation function $\Gamma_{\mu \sigma}$ nested in it, is known to affect the ghost-gluon vertex only by $2 \%$ [148]; thus, its omission is expected to have an insignificant effect on $\mathcal{W}\left(r^{2}\right)$. Therefore, the main uncertainty originates from the term $\mathcal{W}_{2}\left(r^{2}\right)$ of Eq. (B5), and is related to our incomplete knowledge of the form factors $X_{i}$ and $Y_{i}$ for general kinematics.

In this regard, an examination of the integrand of $\mathcal{W}_{2}\left(r^{2}\right)$ in Eq. (B5) shows that this contribution is dominated by the projection $L_{s g}\left(r^{2}\right)$ of the full three-gluon vertex that it contains. In turn, this observation suggests that the SDE determination of $\mathcal{W}\left(r^{2}\right)$ should be fairly accurate provided that the ansatz employed for the general kinematics threegluon vertex reproduces in the soft-gluon limit the $L_{s g}\left(r^{2}\right)$ obtained on the lattice [106].

\section{A closer look at the SDE kernel}

To elucidate this last point, denote by $\mathcal{I}(x, y, \phi)$ the integrand of $\mathcal{W}_{2}(x)$ in Eq. (B5), i.e.,

$\mathcal{I}(x, y, \phi):=s_{\phi}^{4} y \sqrt{x y} \Delta(y) \Delta(u) \frac{F(u)}{u^{2}} B_{1}(u, 0,0) \mathcal{K}(x, y, u)$.
Then, since $F(u)$ and, especially, $\Delta(u)$ are decreasing functions of $u$, the term $\Delta(u) F(u) / u^{2}$ in the second line of Eq. (B7) causes the $\mathcal{I}(x, y, \phi)$ to decrease rapidly at large $u$. Hence, $\mathcal{W}_{2}(x)$ should be dominated by the small $u$ region of its integrand.

Next, recalling that $u=x+y-2 \sqrt{x y} c_{\phi}$, we note that $u=0$ occurs when $y=x$ and $\phi=0$ simultaneously. Also, we emphasize that, in spite of the presence of the factor $u^{2}$ in the denominator, Eq. (B7) is finite at $u=0$, due to the vanishing of $s_{\phi}^{4}$ when $\phi=0$.

In the left panel of Fig. 13 we plot $\mathcal{I}(x, y, \phi)$ for $x=1 \mathrm{GeV}^{2}$ and general $y$ and $\phi$, using the general kinematics $X_{i}$ and $Y_{i}$ of Fig. 11 in the evaluation of $\mathcal{K}(x, y, u)$. There we confirm that $\mathcal{I}(x, y, \phi)$ is largest around $y=x=1 \mathrm{GeV}^{2}$ and $\phi=0$, decaying rapidly to zero at large $u$. Other values of $x$ lead to similar surfaces, with pronounced peaks at $y=x$.

Due to the sharply peaked structure of $\mathcal{I}(x, y, u)$, one expects that the value of the integral defining $\mathcal{W}_{2}(x)$ in Eq. (B5) should depend mainly on the maximum value of $\mathcal{I}(x, y, u)$. To determine the value of this maximum, we expand Eq. (B7) around $y=x$, and finally around $\phi=0 .^{5}$ To this end, first note that

$$
\lim _{\substack{\phi \rightarrow 0 \\ y \rightarrow x}}\left(\frac{s_{\phi}^{4}}{u^{2}}\right)=\frac{1}{x^{2}} .
$$

The limit of the other terms in Eq. (B7) as $u \rightarrow 0$ is straightforward, leading to

\footnotetext{
${ }^{5}$ The limit of $\mathcal{I}(x, y, \phi)$ as $u \rightarrow 0$ is path dependent, vanishing if $\phi=0$ is set first; however, we are interested in its maximum, which occurs when $y=x$ is set first, and $\phi=0$ after, as is clear from Fig. 13.
} 


$$
\begin{aligned}
\lim _{\substack{\phi \rightarrow 0 \\
y \rightarrow x}} \mathcal{I}(x, y, u)= & 3 \Delta(0) F(0) B_{1}(0,0,0) x \Delta(x) \\
& \times\left[X_{1}(x, x, \pi)-x X_{3}(x, x, \pi)\right],
\end{aligned}
$$

which, after using Eq. (A10) becomes

$\lim _{\substack{\phi \rightarrow 0 \\ y \rightarrow x}} \mathcal{I}(x, y, u)=3 \Delta(0) F(0) B_{1}(0,0,0) x \Delta(x) L_{s g}(x)$.

Given that the $X_{i}$ employed reproduce $L_{s g}(x)$ when the combination in Eq. (A10) is formed, the above considerations indicate that our result for $\mathcal{W}\left(r^{2}\right)$ should be rather accurate. Essentially $\mathcal{W}_{2}(x)$ appears dominated by the "slice" that corresponds to $L_{s g}\left(r^{2}\right)$, with little or no effect from all other kinematic configurations.

\section{A special ansatz for the three-gluon vertex}

To confirm this hypothesis explicitly, we compute $\mathcal{W}\left(r^{2}\right)$ using a simpler ansatz for the three-gluon vertex, which also reproduces the limit given in Eq. (B10). Specifically, we substitute the full three-gluon vertex appearing in the second line of Eq. (B2) by

$$
\begin{aligned}
\bar{\Gamma}_{\mu \alpha}^{\mu}(-r, k, t) & \rightarrow \bar{\Gamma}_{0 \mu \alpha}^{\mu}(-r, k, t) \bar{L}_{s g}\left(r^{2}, k^{2}\right), \\
\bar{L}_{s g}\left(r^{2}, k^{2}\right) & =\frac{1}{2}\left[L_{s g}\left(r^{2}\right)+L_{s g}\left(k^{2}\right)\right],
\end{aligned}
$$

where $\bar{\Gamma}_{0}^{\alpha \mu \nu}(q, r, p)$ is the tree-level equivalent of the $\bar{\Gamma}^{\alpha \mu \nu}(q, r, p)$, defined in Eq. (3.3). This ansatz amounts to substituting into Eq. (B6) $X_{1}=X_{4}=X_{7}=\bar{L}_{s g}\left(r^{2}, k^{2}\right)$, with all other $X_{i}$ and $Y_{i}$ set to zero. With this approximation, $\mathcal{W}_{2}(x)$ is still given by Eq. (B5), but with $\mathcal{K}(x, y, u)$ replaced by

$\mathcal{K}(x, y, u) \rightarrow \mathcal{K}_{s g}(x, y, u)=\left[\sqrt{x y}\left(c_{\phi}^{2}+2\right)+u c_{\phi}\right] \bar{L}_{s g}(x, y)$.

Then, substituting Eq. (B12) into Eq. (B7) it is straightforward to show that the limit in Eq. (B10) is exactly reproduced. ${ }^{6}$

The $\mathcal{W}\left(r^{2}\right)$ that is obtained through the use of Eq. (B12), denoted by $\mathcal{W}_{s g}\left(r^{2}\right)$, is shown as the purple dot-dashed curve in the right panel of Fig. 13, where it is compared to the result obtained from Eq. (B4) using the general kinematics $X_{i}$ and $Y_{i}$ (blue solid line). The purple band around $\mathcal{W}_{s g}\left(r^{2}\right)$ corresponds to propagated statistical errors in the $L_{s g}\left(r^{2}\right)$

\footnotetext{
${ }^{6}$ Any combination of the form $\bar{L}_{s g}(x, y)=b L_{s g}(x)+$ $(1-b) L_{s g}(y)$ instead of Eq. (B11) preserves Eq. (B10). The particular form used in (B11) has the advantage of preserving the symmetry of $\mathcal{K}(x, y, u)$ under the exchange of $x \leftrightarrow y$. We have explicitly checked that the extreme cases $b=0$ and $b=1$ lead to results that are nearly identical to those obtained with $b=\frac{1}{2}$, shown in Fig. 13.
}

of [106], obtained by implementing in Eq. (B11) the substitution $L_{s g}\left(r^{2}\right) \rightarrow L_{s g}^{ \pm}\left(r^{2}\right)$ [see Eq. (C13)].

In the right panel of Fig. 13 we see that the two approximations for $\mathcal{W}\left(r^{2}\right)$ agree within the error bands, except for a small region around $3.5 \mathrm{GeV}$. This result indicates that the error in the lattice $L_{s g}\left(r^{2}\right)$ is more important than the detailed general kinematics structure of the full three-gluon vertex, provided the limit in Eq. (B10) is respected.

\section{APPENDIX C: FITS FOR LATTICE INPUTS}

For the gluon and ghost propagators, as well as the threegluon vertex projection $L_{s g}\left(r^{2}\right)$, we employ fits to lattice data $[22,103,106,130]$, appropriately extrapolated to the continuum limit. The fitting functions used incorporate a number of features expected on physical grounds, particularly their asymptotic behaviors for small and large momenta. In particular:

(i) In the UV, they reduce to the one-loop resummed behaviors dictated by renormalization-group arguments, namely

$$
\begin{aligned}
& \lim _{r^{2} \rightarrow \infty} \Delta^{-1}\left(r^{2}\right)=r^{2} L_{\mathrm{UV}}^{\delta}\left(r^{2}\right), \\
& \lim _{r^{2} \rightarrow \infty} F^{-1}\left(r^{2}\right)=L_{\mathrm{UV}}^{\gamma}\left(r^{2}\right), \\
& \lim _{r^{2} \rightarrow \infty} L_{s g}\left(r^{2}\right)=L_{\mathrm{UV}}^{\delta-\gamma}\left(r^{2}\right),
\end{aligned}
$$

where we have defined $L_{\mathrm{UV}}\left(r^{2}\right)=\omega \ln \left(r^{2} / \Lambda^{2}\right)$, with $\omega=11 C_{\mathrm{A}} \alpha_{s} /(12 \pi)$. The anomalous dimensions are given by $\delta=13 / 22$ and $\gamma=9 / 44$.

(ii) $L_{s g}\left(r^{2}\right)$ and the derivative of the gluon propagator diverge logarithmicaly at the origin, i.e.,

$$
\begin{aligned}
\lim _{r^{2} \rightarrow 0} L_{s g}\left(r^{2}\right) & =\ell \ln \left(\frac{r^{2}}{\mu^{2}}\right), \\
\lim _{r^{2} \rightarrow 0}\left[\Delta^{-1}\left(r^{2}\right)\right]^{\prime} & =d \ln \left(\frac{r^{2}}{\mu^{2}}\right),
\end{aligned}
$$

with $\ell$ and $d$ dimensionless constants.

(iii) The $\mathbb{C}\left(r^{2}\right)$ obtained from the BSE is finite at the origin $[79,90,91,93]$. On the other hand, the $L_{s g}(0)$ and $\left[\Delta^{-1}(0)\right]^{\prime}$ appearing in Eq. (4.12) diverge as given by Eq. (C2). Moreover, it can be shown that $\mathcal{W}\left(r^{2}\right)$ has the asymptotic behavior,

$$
\lim _{r^{2} \rightarrow 0} \mathcal{W}\left(r^{2}\right)=\tilde{Z}_{1} w \Delta(0) r^{2} \ln \left(\frac{r^{2}}{\mu^{2}}\right)
$$

with $w$ a dimensionless constant, such that the combination $\mathcal{W}\left(r^{2}\right) / r^{2}$ in Eq. (4.12) is also logarithmicaly divergent at the origin. Consequently, consistency of Eqs. (4.12), (C2), and (C3) with 
the BSE prediction for $\mathbb{C}(0)$ requires that all these logarithmic divergences cancel. Specifically, we demand that

$$
\ell-F(0) \tilde{Z}_{1}(w+d)=0 .
$$

(iv) We adopt the asymmetric MOM renormalization scheme [106-108,108], which imposes that

$$
\Delta^{-1}\left(\mu^{2}\right)=\mu^{2}, \quad F\left(\mu^{2}\right)=1, \quad L_{s g}\left(\mu^{2}\right)=1,
$$

and we take the renormalization point to be $\mu=4.3 \mathrm{GeV}$. The fits for the lattice ingredients are all required to reduce exactly to the above values at $\mu$. In order to incorporate all the above features, the fitting functions have rather elaborate forms.

Starting with $F\left(r^{2}\right)$, an accurate fit to the lattice data is obtained with

$$
F^{-1}\left(r^{2}\right)=A^{\gamma}\left(r^{2}\right)+R\left(r^{2}\right),
$$

where $A\left(r^{2}\right)$,

$$
A\left(r^{2}\right):=1+\omega \ln \left(\frac{r^{2}+\eta^{2}\left(r^{2}\right)}{\mu^{2}+\eta^{2}\left(r^{2}\right)}\right)
$$

with

$$
\eta^{2}\left(r^{2}\right)=\frac{\eta_{1}^{2}}{1+r^{2} / \eta_{2}^{2}}
$$

while $R\left(r^{2}\right)$ is a combination of rational functions,

$$
\begin{aligned}
R\left(r^{2}\right)= & \frac{b_{0}+b_{1}^{2} r^{2}}{1+\left(r^{2} / b_{2}^{2}\right)+\left(r^{2} / b_{3}^{2}\right)^{2}} \\
& -\frac{b_{0}+b_{1}^{2} \mu^{2}}{1+\left(\mu^{2} / b_{2}^{2}\right)+\left(r^{2} / b_{3}^{2}\right)^{2}} .
\end{aligned}
$$

Note that $R\left(r^{2}\right)$ vanishes quickly at infinity, and that $R\left(\mu^{2}\right)=0$ and $A\left(\mu^{2}\right)=1$, enforcing the renormalization condition in Eq. (C5). Moreover, while $A\left(r^{2}\right)$ saturates to a constant at the origin, in the UV it recovers the perturbative logarithm, since $\eta^{2}\left(r^{2}\right) \rightarrow 0$ at large $r^{2}$, such that

$$
\lim _{r^{2} \rightarrow \infty} A\left(r^{2}\right)=L_{\mathrm{UV}}\left(r^{2}\right),
$$

where the function $L_{\mathrm{UV}}\left(r^{2}\right)$ was defined in Eq. (C1) with $\Lambda^{2}=\mu^{2} e^{-1 / \omega}$.

Turning to $\Delta\left(r^{2}\right)$, a form that satisfies all the required conditions is

$\Delta^{-1}\left(r^{2}\right)=r^{2}\left[\frac{d}{1+\left(r^{2} / \kappa^{2}\right)} \ln \left(\frac{r^{2}}{\mu^{2}}\right)+A^{\delta}\left(r^{2}\right)\right]+\nu^{2} R\left(r^{2}\right)$,

where the unprotected logarithm in the first term in brackets describes the IR divergence of $\left[\Delta^{-1}(0)\right]^{\prime}$ and drops out in the $\mathrm{UV}$, while $A\left(r^{2}\right)$ and $R\left(r^{2}\right)$ are given by Eqs. (C7) and (C9). The parameter $\nu=1 \mathrm{GeV}$ serves only to make the dimensionality of $R$ consistent with that of $\Delta^{-1}\left(r^{2}\right)$, without changing the dimensions of the $\sigma_{i}$ parameters in Eq. (C9).

As for $L_{s g}\left(r^{2}\right)$ we use the fitting form

$$
L_{s g}\left(r^{2}\right)=\frac{\ell}{1+\left(r^{2} / \kappa^{2}\right)} \ln \left(\frac{r^{2}}{\mu^{2}}\right)+A^{\delta-\gamma}\left(r^{2}\right)+R\left(r^{2}\right),
$$

with $A\left(r^{2}\right)$ and $R\left(r^{2}\right)$ given by Eqs. (C7) and (C9), respectively.

Note that although in Eqs. (C6), (C11), and (C12) we use the same names for the parameters $\kappa^{2}, \eta_{i}^{2}, b_{0}$, and $b_{i}^{2}$, for economy, they are allowed to assume different values for each of the functions $F\left(r^{2}\right), \Delta\left(r^{2}\right)$, and $L_{s g}\left(r^{2}\right)$.

Next, the coefficient $\ell$ in Eq. (C2), characterizing the rate of divergence of $L_{s g}(0)$, has been determined from lattice results to be $\ell=0.11$ [106], and is held fixed during the fitting procedure. In contrast, the rate of divergence $d$ of $\left[\Delta^{-1}\left(r^{2}\right)\right]^{\prime}$ is not accurately determined from the lattice, since the derivative is sensitive to the larger lattice noise in $\Delta\left(r^{2}\right)$ in the deep IR. Moreover, the coefficient $w$ in
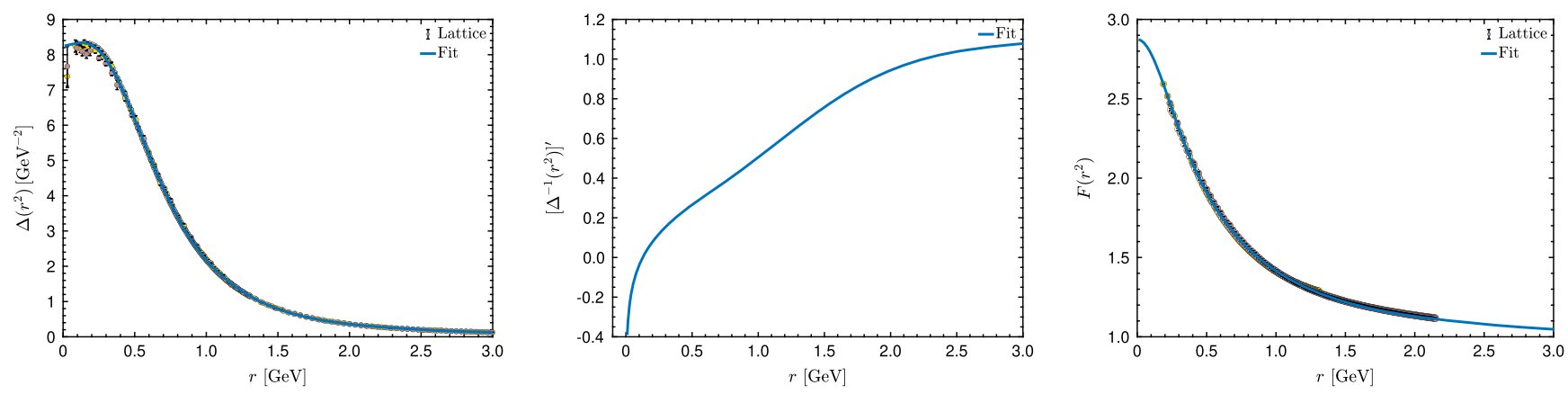

FIG. 14. Left: lattice data of [22,130] (points), and the fit of Eq. (C11) (blue solid line), for the gluon propagator, $\Delta\left(r^{2}\right)$. Center: derivative $\left[\Delta^{-1}\left(r^{2}\right)\right]^{\prime}$, obtained through differentiation of Eq. (C11). Right: lattice data (points) of [103], and the fit of Eq. (C6) (blue solid), for the ghost dressing function, $F\left(r^{2}\right)$. 
TABLE II. Fitting parameters $\kappa^{2}, \eta_{i}^{2}, b_{0}$, and $b_{i}^{2}$, to be used in each of Eqs. (C6), (C11), and (C12) for $F\left(r^{2}\right)$, $\Delta\left(r^{2}\right)$, and $L_{s g}\left(r^{2}\right)$, respectively. In addition to the values below, we set $d=0.075$ and $\ell=0.11$, in Eqs. (C11) and (C12), respectively, and $\omega=0.214$ in the Eqs. (C6), (C11), and (C12).

\begin{tabular}{lccccccc}
\hline \hline & $\kappa^{2}\left[\mathrm{GeV}^{2}\right]$ & $\eta_{1}^{2}\left[\mathrm{GeV}^{2}\right]$ & $\eta_{2}^{2}\left[\mathrm{GeV}^{2}\right]$ & \multicolumn{1}{c}{$b_{0}$} & $b_{1}^{2}\left[\mathrm{GeV}^{-2}\right]$ & $b_{2}^{2}\left[\mathrm{GeV}^{2}\right]$ & $b_{3}^{2}\left[\mathrm{GeV}^{2}\right]$ \\
\hline$F\left(r^{2}\right)$ & - & 3.60 & 0.148 & -0.566 & 0.004 & 0.375 & 24.2 \\
$\Delta\left(r^{2}\right)$ & 1.33 & 0.889 & 2.570 & 1.254 & 0.723 & 1.553 & 2.08 \\
$L_{s g}\left(r^{2}\right)$ & 18.2 & 0.200 & 6.36 & 0.241 & -0.646 & 0.310 & 1.28 \\
\hline \hline
\end{tabular}

Eq. (C3) depends on the ingredients, including $\Delta\left(r^{2}\right)$, used in the SDE evaluation of $\mathcal{W}\left(r^{2}\right)$ through Eqs. (B4) and (B5). As such, in order to enforce Eq. (C4), $w$ and $d$ have to be varied simultaneously, until the cancellation of the divergences has been reached to acceptable precision.

The fitting parameters resulting for $\Delta\left(r^{2}\right), F\left(r^{2}\right)$, and $L_{s g}\left(r^{2}\right)$ are given in Table II and its caption. The resulting curves for $\Delta\left(r^{2}\right)$ and $F\left(r^{2}\right)$ are compared to the lattice data of $[22,130]$ in Fig. 14 , where we also show $\left[\Delta^{-1}\left(r^{2}\right)\right]^{\prime}$. The lattice data of [106] and corresponding fit for $L_{s g}\left(r^{2}\right)$ are shown as the points and black continuous curve, respectively, in the right panel of Fig. 6.

Comparing the curve of $L_{s g}\left(r^{2}\right)$ in Fig. 6 to that of $\left[\Delta^{-1}\left(r^{2}\right)\right]^{\prime}$ in Fig. 14, we see that $\left[\Delta^{-1}\left(r^{2}\right)\right]^{\prime}$ is responsible for reproducing the overall shape of $L_{s g}\left(r^{2}\right)$ in the WI of Eq. (4.12), with the other ingredients providing minor quantitative modulations.
Now, it is clear from Figs. 6 and 14 that the lattice quantity with the largest error in the present analysis is $L_{s g}\left(r^{2}\right)$. In order to propagate the error of $L_{s g}\left(r^{2}\right)$ to other quantities that depend on it, we make a band around $L_{s g}\left(r^{2}\right)$, delimited by

$$
L_{s g}^{ \pm}\left(r^{2}\right)=L_{s g}\left(r^{2}\right) \pm \frac{\delta_{1}}{1+\left(r^{2} / \delta_{2}^{2}\right)^{2}}
$$

with parameters $\delta_{1}=0.08$ and $\delta_{2}^{2}=5 \mathrm{GeV}^{2}$.

Lastly, for the value of $\alpha_{s}$ in the asymmetric MOM scheme, which appears in the SDE for $\mathcal{W}\left(r^{2}\right)$ given by Eq. (B4), we use the value $\alpha_{s}=0.27$ determined by lattice simulations $[101,149]$. For the $F(0)$ appearing in Eq. (4.12) we obtain from Eq. (C6) and Table II the value $F(0)=2.88$.
[1] W. J. Marciano and H. Pagels, Phys. Rep. 36, 137 (1978).

[2] C. D. Roberts and A. G. Williams, Prog. Part. Nucl. Phys. 33, 477 (1994).

[3] R. Alkofer and L. von Smekal, Phys. Rep. 353, 281 (2001).

[4] C. S. Fischer, J. Phys. G 32, R253 (2006).

[5] C. D. Roberts, Prog. Part. Nucl. Phys. 61, 50 (2008).

[6] D. Binosi and J. Papavassiliou, Phys. Rep. 479, 1 (2009).

[7] D. Binosi, L. Chang, J. Papavassiliou, and C. D. Roberts, Phys. Lett. B 742, 183 (2015).

[8] I. C. Cloet and C. D. Roberts, Prog. Part. Nucl. Phys. 77, 1 (2014).

[9] A. C. Aguilar, D. Binosi, and J. Papavassiliou, Front. Phys.(Beijing) 11, 111203 (2016).

[10] D. Binosi, L. Chang, J. Papavassiliou, S.-X. Qin, and C. D. Roberts, Phys. Rev. D 93, 096010 (2016).

[11] D. Binosi, C. Mezrag, J. Papavassiliou, C. D. Roberts, and J. Rodriguez-Quintero, Phys. Rev. D 96, 054026 (2017).

[12] M. Q. Huber, Phys. Rep. 879, 1 (2020).

[13] J. M. Pawlowski, D. F. Litim, S. Nedelko, and L. von Smekal, Phys. Rev. Lett. 93, 152002 (2004).

[14] J. M. Pawlowski, Ann. Phys. (Amsterdam) 322, 2831 (2007).
[15] A. K. Cyrol, M. Mitter, J. M. Pawlowski, and N. Strodthoff, Phys. Rev. D 97, 054006 (2018).

[16] L. Corell, A. K. Cyrol, M. Mitter, J. M. Pawlowski, and N. Strodthoff, SciPost Phys. 5, 066 (2018).

[17] J.-P. Blaizot, J. M. Pawlowski, and U. Reinosa, Ann. Phys. (Amsterdam) 431, 168549 (2021).

[18] A. Cucchieri and T. Mendes, Proc. Sci. LATTICE2007 (2007) 297.

[19] A. Cucchieri and T. Mendes, Phys. Rev. Lett. 100, 241601 (2008).

[20] A. Cucchieri and T. Mendes, Phys. Rev. D 81, 016005 (2010).

[21] I. Bogolubsky, E. Ilgenfritz, M. Muller-Preussker, and A. Sternbeck, Proc. Sci. LATTICE2007 (2007) 290.

[22] I. Bogolubsky, E. Ilgenfritz, M. Muller-Preussker, and A. Sternbeck, Phys. Lett. B 676, 69 (2009).

[23] O. Oliveira and P. Silva, Proc. Sci. LAT2009 (2009) 226.

[24] O. Oliveira and P. Bicudo, J. Phys. G 38, 045003 (2011).

[25] A. Maas, Phys. Rep. 524, 203 (2013).

[26] P. Boucaud, J. P. Leroy, A. L. Yaouanc, J. Micheli, O. Pene, and J. Rodriguez-Quintero, Few-Body Syst. 53, 387 (2012). 
[27] O. Oliveira and P. J. Silva, Phys. Rev. D 86, 114513 (2012).

[28] P. Maris and C. D. Roberts, Phys. Rev. C 56, 3369 (1997).

[29] P. Maris and C. D. Roberts, Int. J. Mod. Phys. E 12, 297 (2003).

[30] J. Braun, H. Gies, and J. M. Pawlowski, Phys. Lett. B 684, 262 (2010).

[31] G. Eichmann, I. C. Cloet, R. Alkofer, A. Krassnigg, and C. D. Roberts, Phys. Rev. C 79, 012202 (2009).

[32] I. Cloet, G. Eichmann, B. El-Bennich, T. Klahn, and C. Roberts, Few-Body Syst. 46, 1 (2009).

[33] Ph. Boucaud, J. P. Leroy, A. Le Yaouanc, J. Micheli, O. Pène, and J. Rodríguez-Quintero, J. High Energy Phys. 06 (2008) 099.

[34] G. Eichmann, R. Alkofer, A. Krassnigg, and D. Nicmorus, Phys. Rev. Lett. 104, 201601 (2010).

[35] L. Fister and J. M. Pawlowski, Phys. Rev. D 88, 045010 (2013).

[36] C. A. Meyer and E. S. Swanson, Prog. Part. Nucl. Phys. 82, 21 (2015).

[37] G. Eichmann, H. Sanchis-Alepuz, R. Williams, R. Alkofer, and C. S. Fischer, Prog. Part. Nucl. Phys. 91, 1 (2016).

[38] H. Sanchis-Alepuz, R. Alkofer, and C. S. Fischer, Eur. Phys. J. A 54, 41 (2018).

[39] R. Alkofer, A. Maas, W. A. Mian, M. Mitter, J. Pars-Lpez, J. M. Pawlowski, and N. Wink, Phys. Rev. D 99, 054029 (2019).

[40] F. Gao, S.-X. Qin, C. D. Roberts, and J. RodriguezQuintero, Phys. Rev. D 97, 034010 (2018).

[41] E. V. Souza, M. N. Ferreira, A. C. Aguilar, J. Papavassiliou, C. D. Roberts, and S.-S. Xu, Eur. Phys. J. A 56, 25 (2020).

[42] S.-S. Xu, Z.-F. Cui, L. Chang, J. Papavassiliou, C. D. Roberts, and H.-S. Zong, Eur. Phys. J. A 55, 113 (2019).

[43] A. C. Aguilar et al., Eur. Phys. J. A 55, 190 (2019).

[44] M. Q. Huber, C. S. Fischer, and H. Sanchis-Alepuz, Eur. Phys. J. C 80, 1077 (2020).

[45] C. D. Roberts and S. M. Schmidt, Eur. Phys. J. ST 229, 3319 (2020).

[46] C. D. Roberts, Symmetry 12, 1468 (2020).

[47] J. Horak, J. Papavassiliou, J. M. Pawlowski, and N. Wink, Phys. Rev. D 104, 074017 (2021).

[48] C. D. Roberts, AAPPS Bull. 31, 6 (2021).

[49] J. Smit, Phys. Rev. D 10, 2473 (1974).

[50] J. M. Cornwall, Phys. Rev. D 26, 1453 (1982).

[51] C. W. Bernard, Phys. Lett. B 108, 431 (1982).

[52] C. W. Bernard, Nucl. Phys. B219, 341 (1983).

[53] J. F. Donoghue, Phys. Rev. D 29, 2559 (1984).

[54] J. Mandula and M. Ogilvie, Phys. Lett. B 185, 127 (1987).

[55] J. M. Cornwall and J. Papavassiliou, Phys. Rev. D 40, 3474 (1989).

[56] K. G. Wilson, T. S. Walhout, A. Harindranath, W.-M. Zhang, R. J. Perry, and S. D. Glazek, Phys. Rev. D 49, 6720 (1994).

[57] O. Philipsen, Nucl. Phys. B628, 167 (2002).

[58] A. C. Aguilar, A. A. Natale, and P. S. Rodrigues da Silva, Phys. Rev. Lett. 90, 152001 (2003).

[59] A. C. Aguilar and A. A. Natale, J. High Energy Phys. 08 (2004) 057.

[60] A. C. Aguilar and J. Papavassiliou, J. High Energy Phys. 12 (2006) 012.
[61] A. C. Aguilar, D. Binosi, and J. Papavassiliou, Phys. Rev. D 78, 025010 (2008).

[62] C. S. Fischer, A. Maas, and J. M. Pawlowski, Ann. Phys. (Amsterdam) 324, 2408 (2009).

[63] M. Tissier and N. Wschebor, Phys. Rev. D 82, 101701 (2010).

[64] D. Binosi, D. Ibañez, and J. Papavassiliou, Phys. Rev. D 86, 085033 (2012).

[65] J. Serreau and M. Tissier, Phys. Lett. B 712, 97 (2012).

[66] M. Peláez, M. Tissier, and N. Wschebor, Phys. Rev. D 90, 065031 (2014).

[67] A. C. Aguilar, D. Binosi, C. T. Figueiredo, and J. Papavassiliou, Phys. Rev. D 94, 045002 (2016).

[68] D. Dudal, J. A. Gracey, S. P. Sorella, N. Vandersickel, and H. Verschelde, Phys. Rev. D 78, 065047 (2008).

[69] J. Rodriguez-Quintero, J. High Energy Phys. 01 (2011) 105.

[70] M. R. Pennington and D. J. Wilson, Phys. Rev. D 84, 119901 (2011).

[71] J. Meyers and E. S. Swanson, Phys. Rev. D 90, 045037 (2014).

[72] F. Siringo, Nucl. Phys. B907, 572 (2016).

[73] A. K. Cyrol, J. M. Pawlowski, A. Rothkopf, and N. Wink, SciPost Phys. 5, 065 (2018).

[74] A. Cucchieri, T. Mendes, and E. M. S. Santos, Phys. Rev. Lett. 103, 141602 (2009).

[75] A. Cucchieri, T. Mendes, G. M. Nakamura, and E. M. S. Santos, Proc. Sci. FACESQCD2010 (2010) 026.

[76] P. Bicudo, D. Binosi, N. Cardoso, O. Oliveira, and P. J. Silva, Phys. Rev. D 92, 114514 (2015).

[77] D. Epple, H. Reinhardt, W. Schleifenbaum, and A. P. Szczepaniak, Phys. Rev. D 77, 085007 (2008).

[78] D. R. Campagnari and H. Reinhardt, Phys. Rev. D 82, 105021 (2010).

[79] A. C. Aguilar, D. Binosi, and J. Papavassiliou, Phys. Rev. D 95, 034017 (2017).

[80] S. D. Glazek, M. Gómez-Rocha, J. More, and K. Serafin, Phys. Lett. B 773, 172 (2017).

[81] P. O. Bowman, U. M. Heller, D. B. Leinweber, M. B. Parappilly, A. Sternbeck, L. von Smekal, A. G. Williams, and J.-B. Zhang, Phys. Rev. D 76, 094505 (2007).

[82] W. Kamleh, P. O. Bowman, D. B. Leinweber, A. G. Williams, and J. Zhang, Phys. Rev. D 76, 094501 (2007).

[83] A. Ayala, A. Bashir, D. Binosi, M. Cristoforetti, and J. Rodriguez-Quintero, Phys. Rev. D 86, 074512 (2012).

[84] A. C. Aguilar, D. Binosi, and J. Papavassiliou, Phys. Rev. D 88, 074010 (2013).

[85] A. C. Aguilar, F. De Soto, M. N. Ferreira, J. Papavassiliou, J. Rodrguez-Quintero, and S. Zafeiropoulos, Eur. Phys. J. C 80, 154 (2020).

[86] J. S. Schwinger, Phys. Rev. 125, 397 (1962).

[87] J. S. Schwinger, Phys. Rev. 128, 2425 (1962).

[88] R. Jackiw and K. Johnson, Phys. Rev. D 8, 2386 (1973).

[89] E. Eichten and F. Feinberg, Phys. Rev. D 10, 3254 (1974).

[90] A. C. Aguilar, D. Ibanez, V. Mathieu, and J. Papavassiliou, Phys. Rev. D 85, 014018 (2012).

[91] D. Ibañez and J. Papavassiliou, Phys. Rev. D 87, 034008 (2013).

[92] D. Binosi and J. Papavassiliou, Phys. Rev. D 97, 054029 (2018). 
[93] A. C. Aguilar, D. Binosi, C. T. Figueiredo, and J. Papavassiliou, Eur. Phys. J. C 78, 181 (2018).

[94] G. Eichmann, J. M. Pawlowski, and J. a. M. Silva, Phys. Rev. D 104, 114016 (2021).

[95] J. Taylor, Nucl. Phys. B33, 436 (1971).

[96] A. Slavnov, Theor. Math. Phys. 10, 99 (1972).

[97] J. Cornwall and R. Norton, Phys. Rev. D 8, 3338 (1973).

[98] E. C. Poggio, E. Tomboulis, and S. H. H. Tye, Phys. Rev. D 11, 2839 (1975).

[99] A. Cucchieri, A. Maas, and T. Mendes, Phys. Rev. D 74, 014503 (2006).

[100] A. Cucchieri, A. Maas, and T. Mendes, Phys. Rev. D 77, 094510 (2008).

[101] A. Athenodorou, D. Binosi, P. Boucaud, F. De Soto, J. Papavassiliou, J. Rodriguez-Quintero, and S. Zafeiropoulos, Phys. Lett. B 761, 444 (2016).

[102] A. G. Duarte, O. Oliveira, and P. J. Silva, Phys. Rev. D 94, 074502 (2016).

[103] P. Boucaud, F. De Soto, K. Raya, J. Rodríguez-Quintero, and S. Zafeiropoulos, Phys. Rev. D 98, 114515 (2018).

[104] P. Boucaud, F. De Soto, A. Le Yaouanc, J. P. Leroy, J. Micheli, H. Moutarde, O. Pene, and J. RodriguezQuintero, J. High Energy Phys. 04 (2003) 005.

[105] P. Boucaud, F. De Soto, A. Le Yaouanc, J. P. Leroy, J. Micheli, O. Pene, and J. Rodriguez-Quintero, Phys. Rev. D 70, 114503 (2004).

[106] A. C. Aguilar, F. De Soto, M. N. Ferreira, J. Papavassiliou, and J. Rodríguez-Quintero, Phys. Lett. B 818, 136352 (2021).

[107] A. C. Aguilar, M. N. Ferreira, and J. Papavassiliou, Eur. Phys. J. C 80, 887 (2020).

[108] A. C. Aguilar, M. N. Ferreira, and J. Papavassiliou, Eur. Phys. J. C 81, 54 (2021).

[109] B. S. DeWitt, Phys. Rev. 162, 1195 (1967).

[110] J. Honerkamp, Nucl. Phys. B48, 269 (1972).

[111] G. 't Hooft, Nucl. Phys. B35, 167 (1971).

[112] R. E. Kallosh, Nucl. Phys. B78, 293 (1974).

[113] H. Kluberg-Stern and J. B. Zuber, Phys. Rev. D 12, 482 (1975).

[114] L. Abbott, Nucl. Phys. B185, 189 (1981).

[115] G. M. Shore, Ann. Phys. (N.Y.) 137, 262 (1981).

[116] L. F. Abbott, M. T. Grisaru, and R. K. Schaefer, Nucl. Phys. B229, 372 (1983).

[117] A. C. Aguilar, D. Binosi, D. Ibañez, and J. Papavassiliou, Phys. Rev. D 89, 085008 (2014).

[118] C. Becchi, A. Rouet, and R. Stora, Ann. Phys. (N.Y.) 98, 287 (1976).

[119] I. V. Tyutin, arXiv:0812.0580.

[120] M. A. L. Capri, D. Fiorentini, M. S. Guimaraes, B. W. Mintz, L. F. Palhares, S. P. Sorella, D. Dudal, I. F. Justo, A. D. Pereira, and R. F. Sobreiro, Phys. Rev. D 92, 045039 (2015).

[121] N. Alkofer and R. Alkofer, Phys. Lett. B 702, 158 (2011).
[122] T. Kugo and I. Ojima, Prog. Theor. Phys. Suppl. 66, 1 (1979).

[123] J. Papavassiliou, Phys. Rev. D 41, 3179 (1990).

[124] A. K. Cyrol, L. Fister, M. Mitter, J. M. Pawlowski, and N. Strodthoff, Phys. Rev. D 94, 054005 (2016).

[125] A. Pilaftsis, Nucl. Phys. B487, 467 (1997).

[126] D. Binosi and J. Papavassiliou, Phys. Rev. D 77, 061702 (2008).

[127] A. C. Aguilar and J. Papavassiliou, Phys. Rev. D 81, 034003 (2010).

[128] D. Binosi and J. Papavassiliou, Phys. Rev. D 66, 025024 (2002).

[129] A. C. Aguilar, M. N. Ferreira, C. T. Figueiredo, and J. Papavassiliou, Phys. Rev. D 99, 034026 (2019).

[130] A. C. Aguilar, C. O. Ambrósio, F. De Soto, M. N. Ferreira, B. M. Oliveira, J. Papavassiliou, and J. RodríguezQuintero, Phys. Rev. D 104, 054028 (2021).

[131] C. Parrinello, Phys. Rev. D 50, R4247 (1994).

[132] B. Alles, D. Henty, H. Panagopoulos, C. Parrinello, C. Pittori, and D. G. Richards, Nucl. Phys. B502, 325 (1997).

[133] C. Parrinello, D. Richards, B. Alles, H. Panagopoulos, and C. Pittori (UKQCD Collaboration), Nucl. Phys. B Proc. Suppl. 63, 245 (1998).

[134] P. Boucaud, J. P. Leroy, J. Micheli, O. Pene, and C. Roiesnel, J. High Energy Phys. 10 (1998) 017.

[135] A. Sternbeck, P.-H. Balduf, A. Kizilersu, O. Oliveira, P. J. Silva, J.-I. Skullerud, and A. G. Williams, Proc. Sci. LATTICE2016 (2017) 349.

[136] M. Vujinovic and T. Mendes, Phys. Rev. D 99, 034501 (2019).

[137] R. Williams, C. S. Fischer, and W. Heupel, Phys. Rev. D 93, 034026 (2016).

[138] A. C. Aguilar, D. Binosi, and J. Papavassiliou, Phys. Rev. D 89, 085032 (2014).

[139] C. S. Fischer and R. Alkofer, Phys. Rev. D 67, 094020 (2003).

[140] A. C. Aguilar and J. Papavassiliou, Phys. Rev. D 83, 014013 (2011).

[141] R. Alkofer, C. S. Fischer, and F. J. Llanes-Estrada, Phys. Lett. B 611, 279 (2005); 670, 460(E) (2009).

[142] D. Zwanziger, Phys. Rev. D 69, 016002 (2004).

[143] F. Gao, J. Papavassiliou, and J. M. Pawlowski, Phys. Rev. D 103, 094013 (2021).

[144] N. Nakanishi, Prog. Theor. Phys. Suppl. 43, 1 (1969).

[145] M. Blank and A. Krassnigg, Comput. Phys. Commun. 182, 1391 (2011).

[146] J. S. Ball and T.-W. Chiu, Phys. Rev. D 22, 2550 (1980); 23, 3085(E) (1981).

[147] A. C. Aguilar, M. N. Ferreira, C. T. Figueiredo, and J. Papavassiliou, Phys. Rev. D 99, 094010 (2019).

[148] M. Q. Huber, Eur. Phys. J. C 77, 733 (2017).

[149] P. Boucaud, F. De Soto, J. Rodríguez-Quintero, and S. Zafeiropoulos, Phys. Rev. D 95, 114503 (2017). 